\title{
Evolution of flame curvature in turbulent premixed Bunsen flames at different pressure levels
}

Ahmad Alqallaf ${ }^{1}$, Markus Klein $^{2, *}$, Cesar Dopazo ${ }^{3}$, Nilanjan Chakraborty $^{1}$

${ }^{1}$ School of Engineering, Newcastle University, Newcastle-Upon-Tyne, NE1 7RU, UK

${ }^{2}$ Bundeswehr University Munich, Department of Aerospace Engineering, Werner-Heisenberg-

Weg 39, 85577 Neubiberg, Germany

${ }^{3}$ School of Engineering and Architecture - Fluid Mechanics Area, University of Zaragoza,

C/Maria de Luna 3, 50018 Zaragoza, Spain

*Corresponding author 


\begin{abstract}
The physical mechanisms underlying the curvature evolution in turbulent premixed Bunsen flames at different thermodynamic pressures are investigated using a three-dimensional Direct Numerical Simulation database. It is found that, due to the occurrence of the Darrieus-Landau instability, the high-pressure flame exhibits higher probability than the low-pressure cases of developing large negative curvature values and saddle concave topologies. The terms in the curvature transport equation due to normal strain rate gradients and curl of vorticity arising from both turbulent flow and flame normal propagation play pivotal roles in the curvature evolution. The mean value of the net contribution of the flame propagation terms dominates over the net contributions arising from the background fluid motion. The net contribution of the source/sink terms tries to reduce the convexity of the flame surface in the positively curved locations. By contrast, the net contribution of the source/sink terms promotes concavity of the flame surface towards the reactants in the negatively curved regions and this effect is particularly strong for the high pressure flame, where the effects of the Darrieus-Landau instability are prominent. This also gives rise to large negative skewness of the probability density functions of curvature in the high-pressure flame with the Darrieus-Landau instability.
\end{abstract}

Keywords: flame curvature; iso-scalar non-material surfaces; turbulent premixed flame 


\section{INTRODUCTION}

The local flame surface topology in premixed flames can be characterised in terms of flame curvature [1]. The importance of flame curvature statistics on turbulent premixed combustion modelling deserves some scrutiny [2,3]. Analytical studies [4-8] indicate that flame curvature significantly affects flame propagation in perturbed laminar premixed flames and plays a key role in thermo-diffusive and hydrodynamic instabilities. The flame curvature has been shown to affect local displacement speed [9-30] and the magnitude of reactive scalar gradient $[19,24,31-35]$ in turbulent premixed flames. Moreover, the differential diffusion of heat and species, arising from non-unity Lewis number, has been found to affect the curvature dependences of displacement speed [18,25,31], consumption speed [36,37] and reactive scalar gradient [24]. Furthermore, Creta et al. [38] postulate that the negative skewness of the probability density function (PDF) of curvature is a marker of the Darrieus-Landau (D-L) instability (an intrinsic flame instability that occurs in premixed flames due to the thermal expansion of the gas) in Bunsen burner flames. Klein et al. [39] demonstrated that the negative skewnesss in the curvature PDF can arise also due to Huygens propagation (i.e., the flame surface propagation normal to itself) and that the skewness of the curvature PDF becomes increasingly negative for Bunsen flames with increasing pressure. They also suggested a criterion for negative skewness based on two alternative mechanisms, i.e. Huygens propagation and the D-L instability. The latter is caused due to large separation between the nozzle diameter (i.e., hydrodynamic length scale) and the flame thickness, such that the range of unstable wavelength exceeds the critical wavelength for D-L instability. This motivates a thorough analysis aiming at understanding the physical mechanisms, which play an important role in the evolution of curvature in turbulent Bunsen burner flames, as well as their pressure dependence and their behaviour under the D-L instability. For more information on the D-L instability the reader is referred to references [38,39] and the literature cited therein. The present analysis 
addresses the aforementioned gaps in the existing literature by studying the statistical behaviours of the terms of the transport equation of local flame curvature.

A transport equation of surface curvature in parametric coordinates was derived by Pope [40]. Recently, Dopazo et al. [41] proposed an alternative derivation in general Cartesian coordinates, which reveals that the normal strain rate and the vorticity, caused by a curved isoscalar surface propagating with a spatially variable displacement speed, significantly affect the evolution of curvature in addition to flow normal strain rates and vorticity. This analysis was subsequently extended by Cifuentes et al. [42] to analyse the curvature transport in a turbulent bluff body burner using a flame-resolved three-dimensional simulation. It has been found that the contributions arising from flame propagation dominates over the flow-induced terms in the curvature transport equation. Neither of these analyses addressed the physical mechanisms which play active role in the curvature distribution characteristic of the DL instabilities (e.g. negative skewness of the curvature probability density functions [38,39]). These effects are yet to be fully understood and this paper addresses this important void in the existing literature. To the best of knowledge of these authors, there is no study available in the open literature where the effects of DL instabilities are analysed in terms of the curvature transport by considering turbulent Bunsen flames for different pressure levels.

By considering the budgets of the various terms in the curvature transport equation, it is possible to establish a relation between the terms of the curvature transport equation and flame instabilities that might occur under certain conditions. This, in turn, enables one to understand if positive or negative flame curvature elements behave neutral, are damped or possibly amplified in the presence of flame instabilities. 
Previous analyses [34,35] indicate that the mean behaviours of dilatation rate and tangential strain rate in Bunsen flames are affected by the occurrence of the DL instability at high pressure, and these strain rate statistics may have a significant role in the curvature evolution at elevated pressure. A DNS database of five different methane-air turbulent premixed Bunsen burner flames has been considered to analyse these issues. Three different Bunsen burner cases (A-C) with different pressures have been considered for a given set of values of root-meansquare turbulent fluctuating velocity normalised with the unstrained laminar burning velocity $u^{\prime} / S_{L}$, normalised bulk mean inlet velocity $U_{B} / S_{L}$ and integral length scale to nozzle diameter ratio $l / D$. For methane-air flames, the laminar burning velocity $S_{L}$ decreases with increasing pressure $P$ according to $S_{L} \sim P^{-0.5}$ [43]. Moreover, the gas density increases proportionally to pressure (i.e., $\rho \propto P$ ), which leads to a reduction of the unburned gas kinematic viscosity with increasing pressure as $v_{u} \sim P^{-1}$. Thus, the thermal flame thickness $\delta_{t h}$ decreases with increasing pressure as $\delta_{t h} \sim v_{u} / S_{L} \sim P^{-0.5}$. Both the turbulent Reynolds number, $R e_{t}=u^{\prime} l / v_{u} \sim u^{\prime} l /$ $\left(S_{L} \delta_{t h}\right)$, and the flow Reynolds number, $R e_{D}=U_{B} D / v_{u} \sim U_{B} D /\left(S_{L} \delta_{t h}\right)$, for cases A-C increase with increasing pressure according to $R e_{t} \sim P^{0.5}$ and $R e_{D} \sim P^{0.5}$, respectively. Hence, cases A-C occupy different locations on the combustion regime diagram [44]. Two additional cases (D and E) at the lowest pressure have been considered with the same turbulent Reynolds number, $R e_{t}$, as that of the highest-pressure case-C. This is achieved by increasing $u^{\prime} / S_{L}$ for a given value of $l / \delta_{t h}$ in case $\mathrm{D}$, whereas $l / \delta_{t h}$ is increased for a given value of $u^{\prime} / S_{L}$ in case $\mathrm{E}$. The main objectives of this investigation are:

- To analyse the effects of pressure on the statistical behaviours of the terms of the curvature transport equation for turbulent premixed Bunsen burner flames.

- To identify the terms and the associated physical mechanisms, which play key roles in the DL instability at high pressure Bunsen flames. 
The mathematical background pertaining to the curvature transport is presented in the next section. A brief description of the numerical implementation follows. Results are next shown and discussed, and, finally, some conclusions are drawn.

\section{MATHEMATICAL BACKGROUND}

The transport equation for the reaction progress variable, $c(\mathbf{x}, t)$, obeys the conservation equation:

$\frac{\partial c}{\partial t}+u_{j} \frac{\partial c}{\partial x_{j}}=\frac{1}{\rho} \frac{\partial}{\partial x_{j}}\left(\rho D_{c} \frac{\partial c}{\partial x_{j}}\right)+\dot{w}_{c}=\frac{1}{\rho} \frac{\partial}{\partial x_{N}}\left(\rho D_{c} \frac{\partial c}{\partial x_{N}}\right)+D_{c} \frac{\partial c}{\partial x_{N}} n_{i, i}+\dot{w}_{c}$

where $u_{j}$ is the $j^{\text {th }}$ component of the flow velocity, $\rho$ is the fluid density, $D_{c}$ is the diffusivity of $c$ and $\dot{w}_{c}$ is chemical reaction rate. The local unit normal vector, $\mathbf{n}$, of a $c$ isosurface, pointing towards the unburned gas side, is defined as: $n_{i}=-\left(\partial c / \partial x_{i}\right) /|\nabla c|=-c_{, i} /|\nabla c|=$ $c_{, i} /\left(\partial c / \partial x_{N}\right)$, where $x_{N}$ is the local coordinate in the normal direction to the isosurface. According to the convention used here, $n_{i, i} / 2=0.5 \partial n_{i} / \partial x_{i}=\kappa_{m}=0.5\left(\kappa_{1}+\kappa_{2}\right)$ is the mean value of the two principal curvatures, $\kappa_{1}$ and $\kappa_{2}$, of the isosurface and will henceforth be referred to as the mean isosurface curvature or, for short, mean curvature. Based on this convention, the flame surface has a positive (negative) curvature where it is convex (concave) toward the reactants. Eq. 1 can be written in the kinematic form for a given $c=c^{*}$ isosurface as [44]:

$\frac{\partial c}{\partial t}+v_{j}^{c} \frac{\partial c}{\partial x_{j}}=\frac{\partial c}{\partial t}+u_{j} \frac{\partial c}{\partial x_{j}}-S_{d}|\nabla c|=0$

The above equation and Eqs. 3-8 are strictly valid for given $c=c^{*}$ isosurface, and this conditional feature is not shown explicitly in these equations in the interests of brevity and readability. Here, $v_{j}^{c}=u_{j}+S_{d} n_{j}$ is the $\mathrm{j}^{\text {th }}$ component of local propagation velocity of a point on a given $c$ isosurface, and $S_{d}$ is the normal displacement speed of a particular isosurface relative to the flow. $S_{d}$ is readily obtained from eqs.1 and $2[11,12,17]$ : 
$S_{d}(\mathbf{x}, t)=\frac{1}{\rho|\nabla c|} \frac{\partial}{\partial x_{N}}\left(\rho D_{c} \frac{\partial c}{\partial x_{N}}\right)-2 D_{c} \kappa_{m}+\frac{\dot{w}_{c}}{|\nabla c|}$

Deriving eq. 1 with respect to $x_{i}$ and setting $c_{, i}=-|\nabla c| n_{i}$, one obtains [41]:

$\frac{1}{|\nabla c|}\left(\frac{\partial|\nabla c| n_{i}}{\partial t}+v_{j}^{c} \frac{\partial|\nabla c| n_{i}}{\partial x_{j}}\right)=-v_{j, i}^{c} n_{j}$

Multiplying (4) by $n_{i}$ and utilising the identities $n_{i} n_{i}=1, n_{i} \partial n_{i} / \partial t=0$ and $n_{i} \partial n_{i} / \partial x_{j}=0$ one gets the following expression by considering the summation implied by repeated indices:

$\frac{1}{|\nabla c|}\left(\frac{\partial|\nabla c|}{\partial t}+v_{j}^{c} \frac{\partial|\nabla c|}{\partial x_{j}}\right)=-n_{i} v_{j, i}^{c} n_{j}$

The quantity $n_{i} v_{j, i}^{c} n_{j}=a_{N}^{c}=a_{N}+\partial S_{d} / \partial x_{N}$ in eq. 5 is the total strain rate normal to the isosurface, $a_{N}=n_{i} S_{i j} n_{j}$ (where $S_{i j}=0.5\left(\partial u_{i} / \partial x_{j}+\partial u_{j} / \partial x_{i}\right)$ is the flow strain rate) is the flow normal strain rate magnitude and $\partial S_{d} / \partial x_{N}$ is an additional normal strain rate due to a displacement speed gradient component perpendicular to the isosurface $[41,45]$. The gradient of an iso-scalar surface local propagation velocity can be expressed as:

$$
\begin{aligned}
v_{j, i}^{c}= & \frac{\partial v_{j}^{c}}{\partial x_{i}}=\frac{1}{2}\left(\frac{\partial v_{j}^{c}}{\partial x_{i}}+\frac{\partial v_{i}^{c}}{\partial x_{j}}\right)+\frac{1}{2}\left(\frac{\partial v_{j}^{c}}{\partial x_{i}}-\frac{\partial v_{i}^{c}}{\partial x_{j}}\right) \\
= & \frac{1}{2}\left(\frac{\partial u_{j}}{\partial x_{i}}+\frac{\partial u_{i}}{\partial x_{j}}\right)+\frac{1}{2}\left(\frac{\partial u_{j}}{\partial x_{i}}-\frac{\partial u_{i}}{\partial x_{j}}\right)+\frac{1}{2}\left(\frac{\partial S_{d}}{\partial x_{i}} n_{j}+\frac{\partial S_{d}}{\partial x_{j}} n_{i}\right)+\frac{1}{2}\left(\frac{\partial S_{d}}{\partial x_{i}} n_{j}-\frac{\partial S_{d}}{\partial x_{j}} n_{i}\right) \\
& +S_{d} \frac{1}{2}\left(\frac{\partial n_{j}}{\partial x_{i}}+\frac{\partial n_{i}}{\partial x_{j}}\right)+S_{d} \frac{1}{2}\left(\frac{\partial n_{j}}{\partial x_{i}}-\frac{\partial n_{i}}{\partial x_{j}}\right)=S_{i j}^{c}-W_{i j}^{c}
\end{aligned}
$$

'The nomenclature associated with terms on the right side of eq. 6 is summarised in Table 1 , where the total strain rate and rotation rate tensors are expressed as $S_{i j}^{c}=S_{i j}+S_{i j}^{a}$ and $W_{j i}^{c}=$ $W_{j i}+W_{j i}^{a}$ respectively $[41,45]$. 


\begin{tabular}{|l|c|}
\hline Description & Term \\
\hline Total strain rate tensor & $S_{i j}^{c}=0.5\left(\partial v_{i}^{c} / \partial x_{j}+\partial v_{j}^{c} / \partial x_{i}\right)$ \\
\hline Total rotation rate tensor & $W_{i j}^{c}=0.5\left(\partial v_{i}^{c} / \partial x_{j}-\partial v_{j}^{c} / \partial x_{i}\right)$ \\
\hline Flow strain rate tensor & $S_{i j}=0.5\left(\partial u_{i} / \partial x_{j}+\partial u_{j} / \partial x_{i}\right)$ \\
\hline Flow rotation rate tensor & $W_{i j}=0.5\left(\partial u_{i} / \partial x_{j}-\partial u_{j} / \partial x_{i}\right)$ \\
\hline Added strain rate tensor & $S_{i j}^{a}=\underbrace{0.5\left[\left(\partial S_{d} / \partial x_{j}\right) n_{i}+\left(\partial S_{d} / \partial x_{i}\right) n_{j}\right]}_{\text {Space dependence of } S_{d}}+\underbrace{S_{d} 0.5\left(\partial n_{i} / \partial x_{j}+\partial n_{j} / \partial x_{i}\right)}_{\text {Propagating curved iso-c }}$ \\
\hline \begin{tabular}{l} 
Added rotation rate \\
tensor $\quad W_{i j}^{a}=\underbrace{0.5\left[\left(\partial S_{d} / \partial x_{j}\right) n_{i}-\left(\partial S_{d} / \partial x_{i}\right) n_{j}\right]}_{\text {Space dependence of } S_{d}}+\underbrace{S_{d} 0.5\left(\partial n_{i} / \partial x_{j}-\partial n_{j} / \partial x_{i}\right)}_{\text {Propagating curved iso-c }}$ \\
\hline
\end{tabular} \\
\hline
\end{tabular}

Table 1: Nomenclature associated with the velocity gradient tensor $v_{i, j}^{c}$ of an isosurface element.

Using eqs. 4 and 5 one gets:

$$
\frac{\partial n_{i}}{\partial t}+v_{j}^{c} \frac{\partial n_{i}}{\partial x_{j}}=-\left(\delta_{i j}-n_{i} n_{j}\right) S_{j k}^{c} n_{k}+W_{i j}^{c} n_{j}
$$

The strain rate $S_{i j}$ and rotation rate $W_{i j}$ tensors arise due to the fluid motion, and the additional strain rate $S_{i j}^{a}$ and rotation $W_{i j}^{a}$ rate tensors originate due to the motion of curved isosurfaces relative to the flow with spatially dependent $S_{d}$. The first term on the right side of eq. 7 is the projection of the strain vector $-\boldsymbol{S}^{c} \cdot \boldsymbol{n}$ on a plane perpendicular to $\boldsymbol{n}$, whereas the second, $W_{i j}^{c} n_{j}=$ $\frac{1}{2} \varepsilon_{i j k} \omega_{j}^{c} n_{k}$, represents the solid body rotation of $\mathbf{n}$ with an angular velocity $\frac{1}{2} \boldsymbol{\omega}^{c}$ (where $\boldsymbol{\omega}^{\mathrm{c}}=$ $\nabla \times \mathbf{v}^{\mathrm{c}}=\nabla \times \mathbf{u}+\nabla \times\left(\mathrm{S}_{\mathrm{d}} \mathbf{n}\right), \boldsymbol{\omega}=\nabla \times \mathbf{u}$ is the flow vorticity and $\boldsymbol{\omega}^{a}=\nabla \times\left(\mathrm{S}_{\mathrm{d}} \mathbf{n}\right)$ is the additional vorticity).

Derivation of eq. 7 with respect to $x_{i}$ yields a transport equation for the mean curvature, $\kappa_{m},[41]:$ 


$$
\begin{aligned}
& \frac{\partial \kappa_{m}}{\partial t}+v_{j}^{c} \frac{\partial \kappa_{m}}{\partial x_{j}}=\underbrace{\underbrace{\frac{a_{N}\left(n_{i, i}\right)}{2}}_{T_{1}}+\underbrace{\frac{1}{2} \frac{\partial a_{N}}{\partial x_{N}}}_{T_{2}} \underbrace{-S_{i j} n_{j, i}}_{T_{3}} \underbrace{-\frac{1}{2} \frac{\partial S_{i j}}{\partial x_{i}} n_{j}}_{T_{4}}+\underbrace{\frac{1}{2} \frac{\partial W_{i j}}{\partial x_{i}} n_{j}}_{T_{5}}}_{\text {flow terms }} \\
& +\underbrace{\underbrace{\frac{1}{2} \frac{\partial S_{d}}{\partial x_{N}} n_{i, i}}_{T_{6}}+\underbrace{\frac{1}{2} \frac{\partial^{2} S_{d}}{\partial x_{N}{ }^{2}}}_{T_{7}} \underbrace{-S_{i j}^{a} n_{j, i}}_{T_{8}} \underbrace{-\frac{1}{2} \frac{\partial S_{i j}^{a}}{\partial x_{i}} n_{j}}_{T_{9}}+\underbrace{\frac{1}{2} \frac{\partial W_{i j}^{a}}{\partial x_{i}} n_{j}}_{T_{10}}}_{\text {added terms }}
\end{aligned}
$$

The terms on the right side of the curvature transport equation (eq. 8) can be split into: i) terms arising from the fluid flow $\left(T_{1-5}\right)$, and ii) terms originating from molecular mixing and chemical reaction $\left(T_{6-10}\right)$. Positive (negative) contributions of the terms on the right side tend to increase the convexity (concavity) of isosurfaces. The physical descriptions of the terms on the right side of eq. 8 are summarised in Table 2.

\begin{tabular}{|c|l|c|l|}
\hline \multicolumn{2}{|c|}{ Flow Terms } & \multicolumn{2}{c|}{ Added Terms } \\
\hline Terms & Contribution & Terms & Contribution \\
\hline$T_{\mathbf{1}}$ & $\begin{array}{l}\text { due to curvature and flow normal strain } \\
\text { rate correlation }\end{array}$ & $T_{\mathbf{6}}$ & $\begin{array}{l}\text { due to curvature and added normal } \\
\text { strain rate correlation }\end{array}$ \\
\hline$T_{\mathbf{2}}$ & $\begin{array}{l}\text { due to flow normal strain rate normal } \\
\text { variation }\end{array}$ & $T_{\mathbf{7}}$ & $\begin{array}{l}\text { due to added normal strain rate normal } \\
\text { variation }\end{array}$ \\
\hline$T_{\mathbf{3}}$ & caused by flow stretching & $T_{\mathbf{8}}$ & caused by added stretching \\
\hline$T_{\mathbf{4}}$ & caused by flow strain rate gradients & $T_{\mathbf{9}}$ & caused by added strain rate gradients \\
\hline$T_{\mathbf{5}}$ & caused by flow vorticity curl & $T_{\mathbf{1 0}}$ & caused by added vorticity curl \\
\hline
\end{tabular}

Table 2: Description of the various terms in the mean curvature transport eq. 8.

\section{NUMERICAL IMPLEMENTATION}

The simulations used for this analysis have been performed using a compressible DNS code called SENGA $[34,35,46]$ where the conservation equations of mass, momentum, energy and reaction progress variables are solved in non-dimensional form. The spatial discretisation uses a $10^{\text {th }}$ order central difference scheme but the order of accuracy gradually drops to a $2^{\text {nd }}$ order 
one-sided scheme at the non-periodic boundaries. The time-advancement is conducted using a low-storage $3^{\text {rd }}$ order Runge-Kutta scheme. The inlet values of flow Reynolds number $R e=$ $U_{B} D / v_{u}$ based on the bulk inlet velocity $U_{B}$, nozzle diameter $D$, and the kinematic viscosity in the unburned gas $v_{u}$, turbulent Reynolds number, $R e_{t}=u^{\prime} l / v_{u}$, normalised root-mean-square (rms) turbulent velocity fluctuation, $u^{\prime} / S_{L}$, normalised inlet velocity $U_{B} / S_{L}$, longitudinal integral length scale to thermal flame thickness ratio, $l / \delta_{t h}$, longitudinal integral length scale to nozzle diameter ratio $l / D$, Damköhler number, $D a=l S_{L} / \delta_{t h} u^{\prime}$, and Karlovitz number, $K a=\left(u^{\prime} / S_{L}\right)^{3 / 2}\left(l / \delta_{t h}\right)^{-1 / 2}$ are given in Table 3. These cases are taken from a large database involving 24 Bunsen flames [39] and 5 weakly turbulent (i.e. small values of $u^{\prime} / S_{L}$ ) cases have been chosen for the current analysis so that the effects of DL instability are clearly evident because turbulence acts to eclipse the effects of DL instability for large values of $u^{\prime} / S_{L}$. Turbulent inflow data has been generated using a modified version of the digital filtering approach. A detailed description can be found in Klein et al. [34].

\begin{tabular}{|c|c|c|c|c|c|c|c|c|c|}
\hline Case & $\boldsymbol{P} / \boldsymbol{P}_{\mathbf{0}}$ & $\boldsymbol{R e}_{\boldsymbol{D}}$ & $\boldsymbol{R e}_{\boldsymbol{t}}$ & $\boldsymbol{U}_{\boldsymbol{B}} / \boldsymbol{S}_{\boldsymbol{L}}$ & $\boldsymbol{u}_{\text {inlet }}^{\prime} / \boldsymbol{S}_{\boldsymbol{L}}$ & $\boldsymbol{l} / \boldsymbol{\delta}_{\boldsymbol{t h}}$ & $\boldsymbol{l} / \boldsymbol{D}$ & $\boldsymbol{K a}$ & $\boldsymbol{D a}$ \\
\hline $\mathrm{A}$ & 1.0 & 399 & 13.30 & 6.0 & 1.0 & 5.20 & $1 / 5$ & 0.45 & 5.00 \\
\hline $\mathrm{B}$ & 5.0 & 892 & 29.26 & 6.0 & 1.0 & 11.40 & $1 / 5$ & 0.30 & 11.36 \\
\hline $\mathrm{C}$ & 10.0 & 1262 & 41.22 & 6.0 & 1.0 & 16.13 & $1 / 5$ & 0.25 & 16.13 \\
\hline $\mathrm{D}$ & 1.0 & 399 & 41.22 & 6.0 & 3.1 & 5.20 & $1 / 5$ & 2.40 & 1.61 \\
\hline $\mathrm{E}$ & 1.0 & 399 & 41.22 & 6.0 & 1.0 & 16.13 & $3 / 5$ & 0.25 & 16.14 \\
\hline
\end{tabular}

Table 3: Attributes of inlet turbulence for the cases considered here

The current analysis considers a generic single-step Arrhenius type irreversible chemical mechanism for the purpose of computational economy due to the demands of flame resolution at high pressures. It is evident from Eq. 8 that curvature transport depends mainly on the statistics of fluid velocity/vorticity, scalar gradient and displacement speed. It has been demonstrated in the past clearly and without any doubt that displacement speed statistics from 
simple chemistry $[17,18,23,25,32]$ and detailed chemistry [11-16] DNS are qualitatively similar. The same is true for the statistics of the reactive scalar gradient obtained from simple chemistry $[31,32,47]$ and detailed chemistry $[24,47]$ DNS. Moreover, the vorticity and subgrid flux statistics obtained from simple chemistry DNS [48-50] are found to be qualitatively consistent with those obtained from detailed chemistry DNS [51,52]. Furthermore, it has been found that models developed based on simple chemistry data $[50,53,54]$ perform equally well in the context of detailed chemistry and transport $[52,55,56]$. The focus of the present work is neither ignition nor emissions but on fundamental turbulence/chemistry interaction and this can be captured at least in a qualitative sense with the help of simple chemistry. Finally it is important to understand, that laminar burning velocity and flame thickness typically (i.e. in hydrocarbon-air mixtures) decrease with increasing pressure and thus flame resolution for a fixed geometry (as in this work) becomes a real challenge for high pressure DNS and nearly impossible in the context of detailed chemistry and transport. Often DNS studies are done for statistically planar flames in a box and the computational domain shrinks with increasing pressure [57]. In this sense the present configuration is a relatively complex geometry for highpressure combustion.

It can be seen from Table 3 that cases $\mathrm{A}, \mathrm{D}$ and $\mathrm{E}$ are atmospheric flames with $P=P_{0}=1.0$ bar, whereas cases $\mathrm{B}$ and $\mathrm{C}$ are flames corresponding to $P=5 P_{0}=5$ bar and $P=10 P_{0}=10$ bar respectly. In the context of a simple chemical mechanism, the pre-exponential factor and kinematic viscosity have been altered to account for the desired pressure dependence of $S_{L}, \delta_{t h}, v$ as discussed in section 1 . The heat release parameter, $\tau=\left(T_{a d}-T_{0}\right) / T_{0}$, and the Zel'dovich number, $\beta=T_{a c}\left(T_{a d}-T_{0}\right) / T_{a d}^{2}$, are taken to be 4.5 and 6.0, respectively, where $T_{a c}$ is the activation temperature. Standard values of Prandtl number $(\operatorname{Pr}=0.7)$ and ratio of specific heats $\left(\gamma_{g}=1.4\right)$ have been used. 
Table 3 shows that cases A-C have identical inlet values of $u^{\prime} / S_{L}, U_{B} / S_{L}$ and $l / D$, but the values of $R e_{t}, D a$ and $K a$ are different. The cases A-C and $\mathrm{E}$ fall on the boundary of the wrinkled and the corrugated flamelets regimes according to the combustion diagram of Peters [44]. The turbulent Reynolds number $R e_{t}$ increases from case A to case $\mathrm{C}$. Furthermore, Table 3 shows that cases $\mathrm{C}, \mathrm{D}$ and $\mathrm{E}$ have same values of $R e_{t}$ but cases $\mathrm{D}$ and $\mathrm{E}$ have one tenth of the pressure of that of case $\mathrm{C}$. The value of $u^{\prime} / S_{L}$ is higher in case $\mathrm{D}$ than in case $\mathrm{C}$ and $\mathrm{E}$, whereas $u^{\prime} / S_{L}$ and $l / \delta_{t h}$ values are exactly the same for cases $\mathrm{C}$ and $\mathrm{E}$ and thus they fall on the same point on the regime diagram. The simulation domain is taken to be $2 D \times 2 D \times 2 D$ corresponding to a cube of $50 \delta_{t h} \times 50 \delta_{t h} \times 50 \delta_{t h} \quad\left[112 \delta_{t h} \times 112 \delta_{t h} \times 112 \delta_{t h}\right]$ $\left(159 \delta_{t h} \times 159 \delta_{t h} \times 159 \delta_{t h}\right)$ for cases A, D and E [case B] (case C), which is discretised using a uniform Cartesian grid of $250 \times 250 \times 250[560 \times 560 \times 560](795 \times 795 \times 795)$ points. The grid used here ensures resolution of both the Kolmogorov length scale and flame thickness.

The boundary opposite to the inlet and lateral boundaries are considered to be partially nonreflecting and they are specified according to the Navier Stokes Characteristic Boundary Conditions (NSCBC) technique [58]. The reacting scalar field is initialised by an unstrained premixed laminar flame solution with the geometry of a hemisphere located at the inflow with its centre coinciding with that of the nozzle. The mean velocity profile after the nozzle exit has been approximated by a "hyperbolic-tangent like" distribution. The velocity fluctuations are constant within the nozzle core and damped to zero within the boundary layer. The choice of the velocity fluctuation profile is motivated by the experimental configuration of Kobayashi et al. [59], where perforated plates are uses as turbulence generators. Further information on the numerical implementation of the inlet boundary condition can be found in Refs. [34, 35, 39]. The statistics presented in this paper have been extracted after twice the mean residence time 
(i.e., $2 L / U_{\text {mean }}$, where $L$ is the axial length of the simulation domain) and at least 10 different realisations have been considered to extract curvature statistics reported in the next section.

\section{RESULTS \& DISCUSSION}

\subsection{Curvature characterisation}

The isosurface of the reaction progress variable $c=0.8$ coloured by normalised Gauss curvature $\kappa_{g} \times \delta_{t h}^{2}=\kappa_{1} \kappa_{2} \times \delta_{t h}^{2}$ (left) and mean curvature $\kappa_{m} \times \delta_{t h}=0.5\left(\kappa_{1}+\kappa_{2}\right) \times \delta_{t h}$ (right) for cases A-E are shown in Fig. 1 which depicts a likelihood of finding concave flame elements much higher than that of convex flame surfaces. High-pressure flames, B and C, are characterised by small magnitudes of $\kappa_{m}$, namely, flat and locally concave and convex patches of the isosurface. Small magnitudes of $\kappa_{g}$ predominate for cases B and C, showing mainly parabolic tile-like patches, with a low presence of elliptic (cup) and hyperbolic (saddle) elements both concave and convex. Parabolic, elliptic and hyperbolic patches are equally probable for flames $\mathrm{A}$ and $\mathrm{E}$ at atmospheric pressure, while for case $\mathrm{D}$ elliptic and hyperbolic surface elements dominate. Differences between the flame surface geometries for cases A-E and $\mathrm{D}$ should be attributed mainly to differences in inlet $u^{\prime} / S_{L}$, which also imply different Karlovitz and Damköhler numbers. 


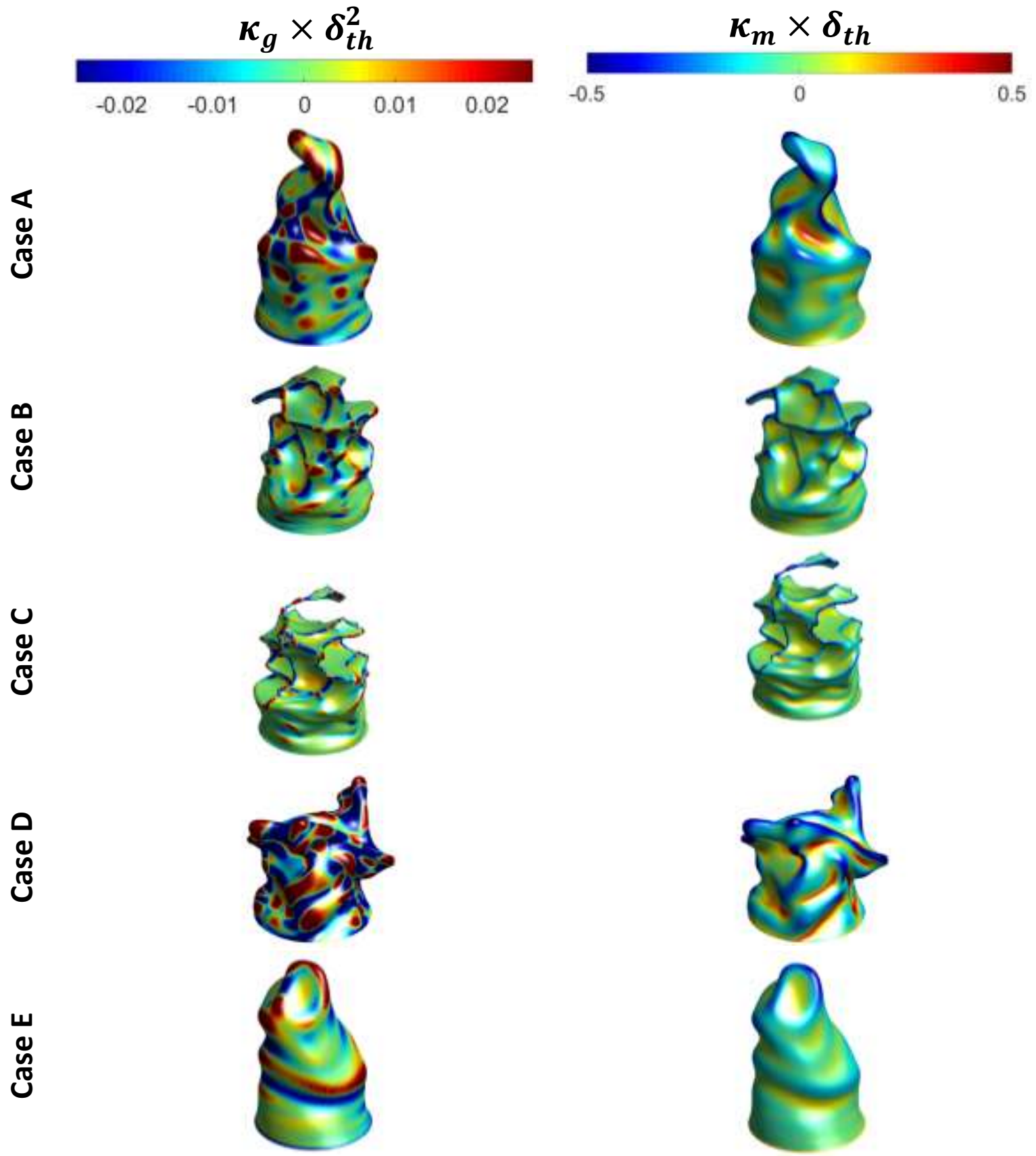

Figure 1: Instantaneous isosurfaces of reaction progress variable $c=0.8$ colored by $\kappa_{g} \times \delta_{t h}^{2}$ (left) and $\kappa_{m} \times \delta_{t h}$ (right) for cases A-E ( $1^{\text {st }}-5^{\text {th }}$ row).

It is worth noting that the surface colour represents normalised curvature values (i.e., $\kappa_{g} \times \delta_{t h}^{2}$ (left) and $\kappa_{m} \times \delta_{t h}$ (right)) and hence agrees qualitatively but not necessarily with the visual impression of flame wrinkling because the flame thickness $\delta_{t h}$ changes with pressure (i.e., $\left.\delta_{t h} \sim P^{-0.5}\right)$. The PDFs of $\kappa_{m} \times \delta_{t h}$ for the $c=0.5$ isosurface are exemplarily shown in Fig. 2 . 
It can be seen from Fig. 2 that the curvature PDFs for the $c=0.5$ isosurface are skewed towards negative values of $\kappa_{m}$, with long tails for $\kappa_{m}<0$ and a qualitatively similar behaviour has been observed for other $c$ isosurfaces. However, it is worth noting that the flame geometry has an additional contribution to the negative values of mean curvature. Although all cases show predominance of negative curvatures, the PDFs of $\kappa_{m}$ display broader distribution in case D than in the other flames, due to greater extent of flame wrinkling in this case as a result of the highest inlet rms velocity $u^{\prime}$ amongst the cases considered here. The skewnesses of the these $\kappa_{m}$ PDFs for cases A-E are given by $-0.80,-1.31,-1.58,-0.33,-0.72$ [39]. While the PDFs of curvature for cases A-C and E show considerable negative skewness, their peak value shifts toward slightly positive $\kappa_{m}$ for the high pressure case $\mathrm{C}$ with greater negative skewness in comparison to low pressure flames A, D and E. It has been discussed elsewhere $[38,39]$ that large negative skewness of the PDFs of $\kappa_{m}$ in the high-pressure cases B and C is indicative of the D-L instability. This can further be discerned from a visual inspection of Fig. 1 where sharply negatively curved flame elements are visible between moderately positively curved bulges in case $\mathrm{C}$ and to a smaller extent in case B. These sharply curved elements between positively curved bulges in cases $\mathrm{B}$ and $\mathrm{C}$ are manifestations of the D-L instability in these high-pressure flames. These positive bulges shift the PDF peak right for both cases B and C. In cases $\mathrm{A}$ and $\mathrm{E}$ the negative skewness of curvature is the artefact of the burner configuration (note that even a laminar Bunsen flame is characterised by negative skewness) and Huygens propagation. Interested readers are referred to Ref. [39] for further information on curvature skewness and D-L instability in these flames. 


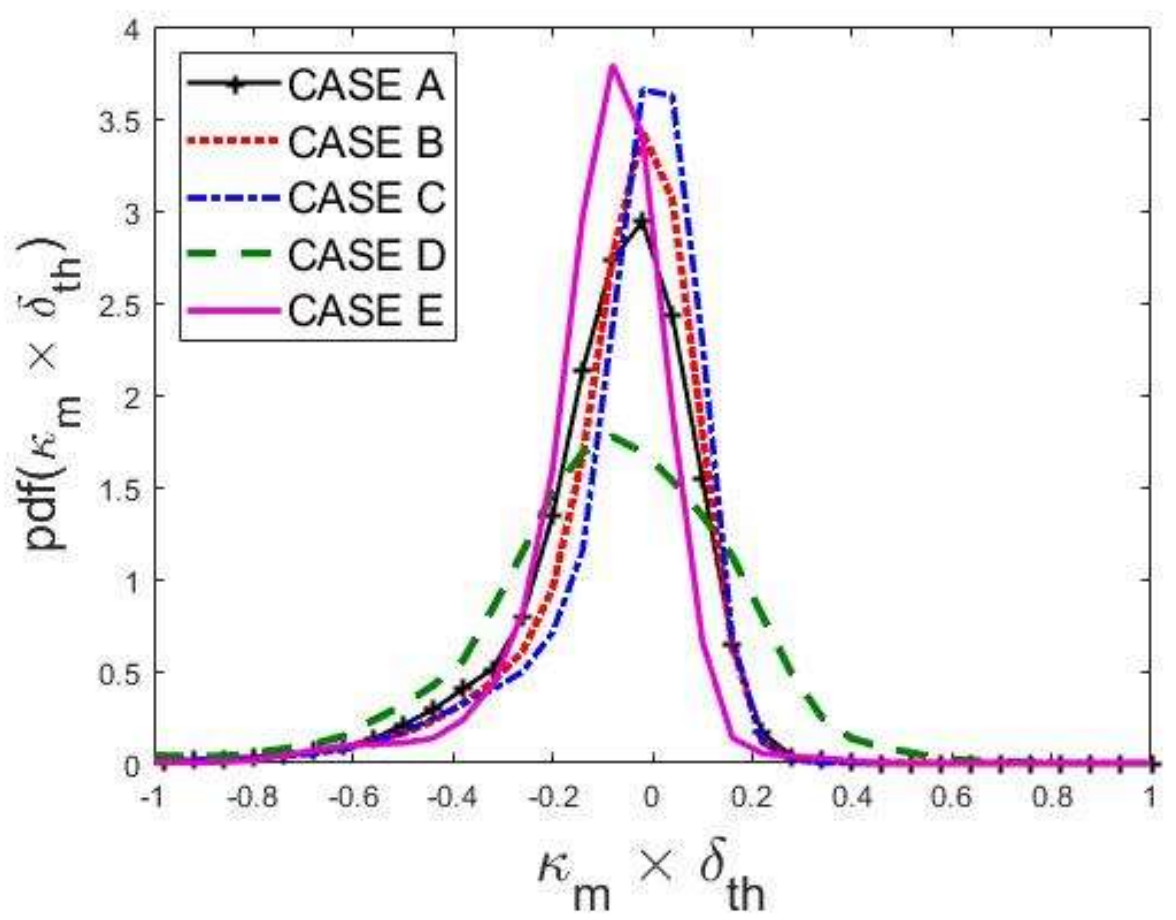

Figure 2: PDFs of $\kappa_{m} \times \delta_{t h}$ on $c=0.5$ isosurface for cases A-E. 

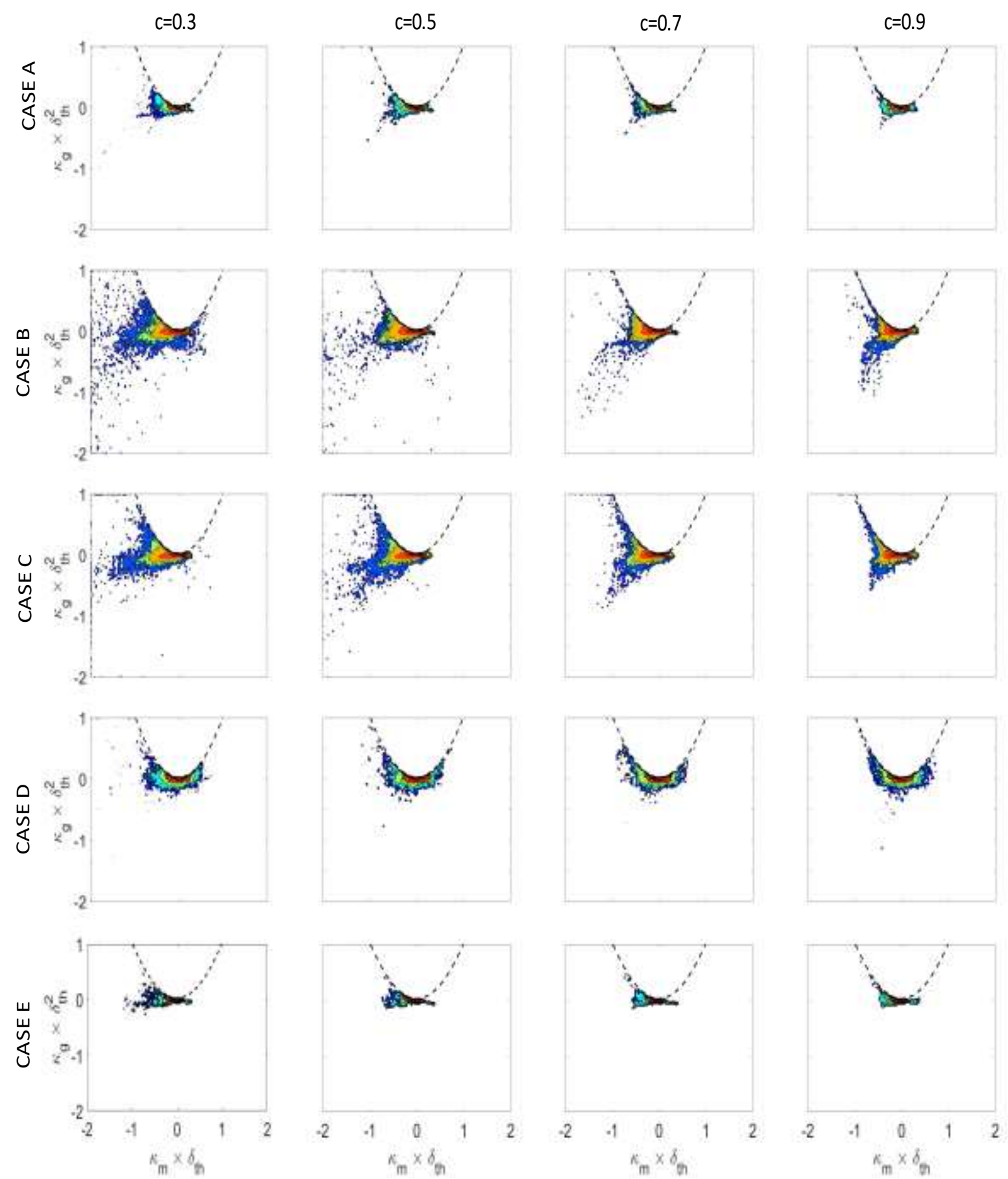

Figure 3: Joint pdf (coloured from blue to dark red) between normalised curvature $\kappa_{m} \times \delta_{t h}$ and normalised Gauss curvature $\kappa_{g} \times \delta_{t h}^{2}$ for $c=0.3,0.5,0.7$ and $0.9\left(1^{\text {st }}-4^{\text {th }}\right.$ column $)$ isosurfaces for cases A-E $\left(1^{\text {st }}-5^{\text {th }}\right.$ row $)$. 
The joint PDF for $\kappa_{g} \times \delta_{t h}^{2}$ and $\kappa_{m} \times \delta_{t h}$ for different $c$ isosurfaces across the flame are shown in Fig. 3. In the $\kappa_{m}-\kappa_{g}$ plane, complex non-physical curvatures are obtained for the region $\kappa_{g}>\kappa_{m}^{2}$. The region $\kappa_{m}>0\left(\kappa_{m}<0\right)$ and $\kappa_{g}>0$ represents cup convex (cup concave) flame topology, whereas $\kappa_{m}>0\left(\kappa_{m}<0\right)$ and $\kappa_{g}<0$ corresponds to saddle convex (saddle concave) flame topology. The combination of $\kappa_{m}>0\left(\kappa_{m}<0\right)$ and $\kappa_{g}=0$ is representative of tile convex (tile concave) flame topology. The probability of finding $\kappa_{m}<0$ dominates over the probability of obtaining $\kappa_{m}>0$ for all Bunsen cases considered here. The concave cup topology (i.e., $\kappa_{m}<0, \kappa_{g}>0$ ) is predominantly obtained for all cases. Furthermore, flat surface topologies (i.e., $\kappa_{m} \approx 0$ and $\kappa_{g} \approx 0$ ) are the most probable ones. The peak value of the $\kappa_{m}-\kappa_{g}$ joint PDF is obtained for slightly positive values of $\kappa_{m}$ for cases B and C, which is consistent with the marginal PDFs of $\kappa_{m}$ for these cases (see Fig. 2). The probability of finding saddle concave topology remains relatively small for cases A, D and E but cases B and C show significant occurrence of locally concave saddle patches. The probability of finding negatively curved elements is higher in case $\mathrm{C}$ than in cases $\mathrm{A}, \mathrm{D}$ and $\mathrm{E}$ due to the presence of the D-L instability, which is reflected in the higher probability of finding saddle concave topologies in case $\mathrm{C}$. The same explanation holds true for the higher presence of saddle concave topologies in case B than in flames A, D and E, but the effects of hydrodynamic instabilities are less prominent in case $\mathrm{B}$ than in case $\mathrm{C}$. The flame $\mathrm{D}$ has the highest $u^{\prime} / S_{L}$ and a characteristic chemical time 2.40 times the Kolmogorov time micro-scale; it represents the thin reaction zone combustion regime, where energetic turbulent eddies cause significant flame distortions. This eventually gives rise to significant probabilities of finding both cup concave and cup convex topologies in case D. Furthermore, the large extent of flame distortion in case D is reflected in the highest probability of obtaining locally positive curvature values and cup convex topologies amongst the cases considered here. 
The budgets of the various terms in the curvature transport equation conditional on $c$ will be discussed in the next section. Based on this analysis, it is possible to judge the magnitude of the different terms of the curvature transport equation and to identify the terms which possibly balance each other. Beside this, another focus of this paper is to establish a relation between the terms of the curvature transport equation and flame instabilities that might occur under certain conditions. It will be therefore instructive to analyse the terms $T_{1}, \ldots, T_{10}$ conditional on mean curvature in subsection 4.5 . Only by this additional representation it becomes possible to understand if positive or negative flame curvature elements behave neutral, are damped or possibly amplified in the presence of flame instabilities.

\subsection{Flow-induced source/sink terms of the curvature transport equation}

The normalised mean values of the flow terms $\left(T_{1-5} \times \delta_{t h}^{2} / S_{L}\right)$ in the curvature transport equation, conditional upon $c$, for flames A-E are shown in Figs. 4a-e. Both qualitative and quantitative behaviours of the terms of the curvature transport equation do not change with axial distance from the nozzle outlet and thus are not shown for the purpose of brevity. Hence, samples for these figures are taken over the whole flame.

The term $T_{1}$, arising from the correlation between normal strain rate and mean curvature, assumes small negative values throughout the flame front. It has been shown elsewhere [34] that the normal strain rate $a_{N}$ exhibits positive values throughout the flame front, whereas the mean value of $\kappa_{m}$ remains negative with small magnitude due to the flame configuration in Bunsen burner flames. The correlation between $a_{N}$ and $\kappa_{m}$ remains weak throughout the flame for the cases considered here [34] and, thus, the combination of predominantly positive $a_{N}$ and negative $\kappa_{m}$ yields negative mean values of $T_{1}$ in cases A-E. 


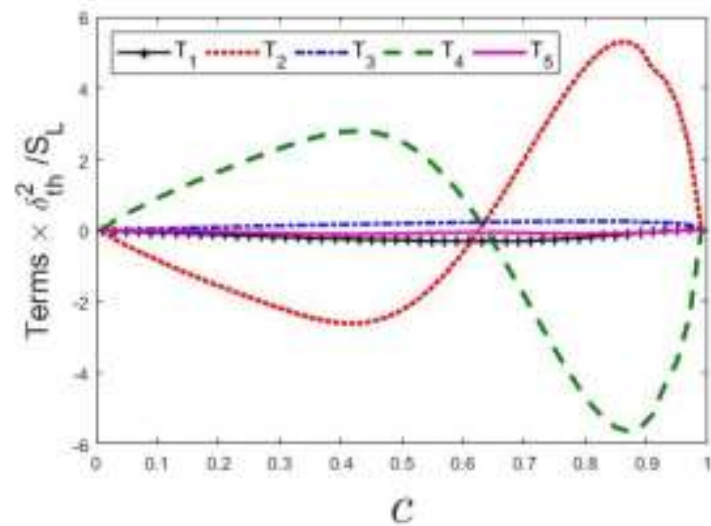

(a)

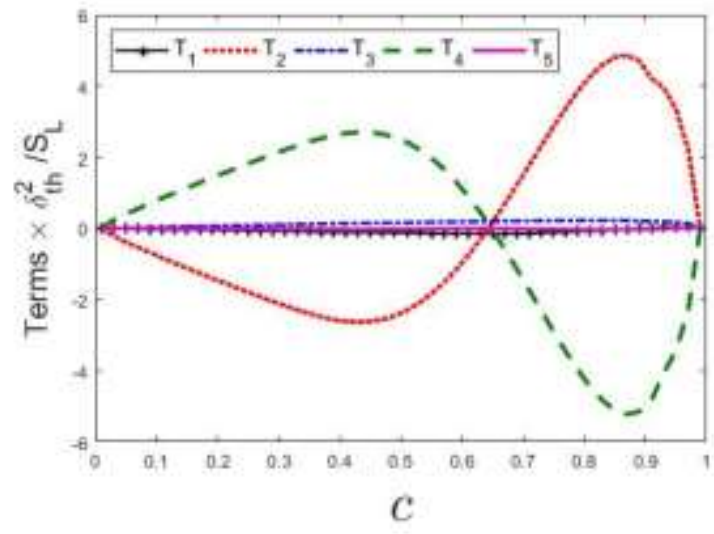

(c)

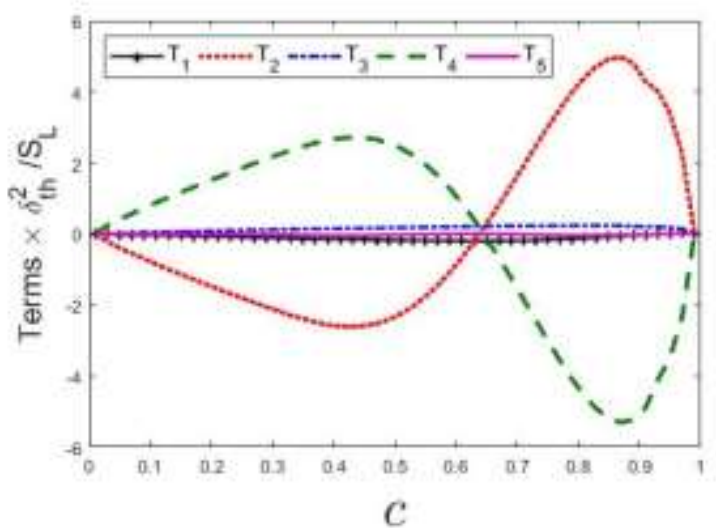

(b)

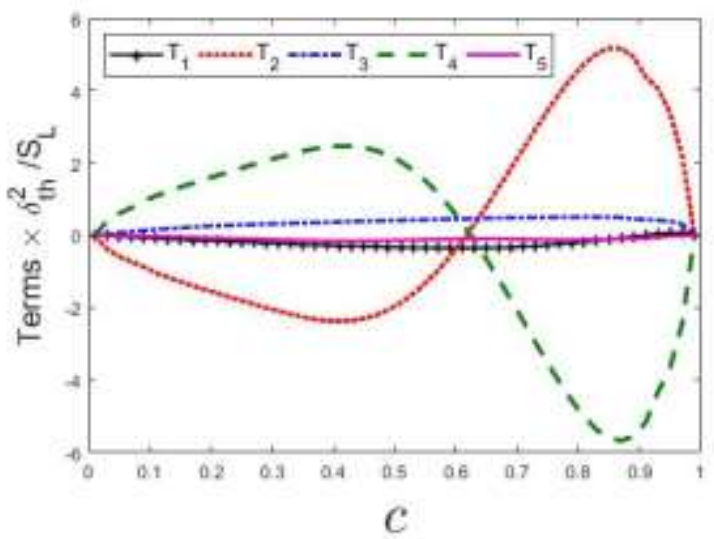

(d)

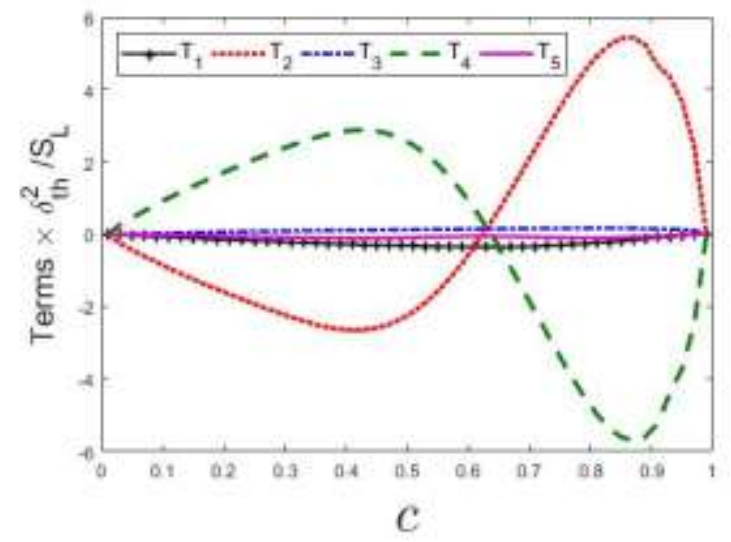

(e)

Figure 4: Profiles of the normalised mean values of the fluid flow induced terms (i.e. $T_{1-5} \times$ $\delta_{t h}^{2} / S_{L}$ ) of the curvature transport equation conditional upon $c$ for cases (a-e) A-E.

The mean value of $T_{2} \times\left(\delta_{t h}^{2} / S_{L}\right)=\left[\left(\partial a_{N} / \partial x_{N}\right) / 2\right] /\left(\delta_{t h}^{2} / S_{L}\right)$, due to the normal derivative of the flow normal strain rate, remains negative on the unburnt side but becomes positive 
towards the burned gas side of the flame. For cases A-E, the mean normal strain rate $a_{N}$ remains positive throughout the flame and assumes a maximum at a location slightly skewed towards the burned gas side of the flame. This leads to predominance of $\left(\partial a_{N} / \partial x_{N}\right)<0$ on the unburned gas side, whereas this behaviour reverses (i.e., $\left.\left(\partial a_{N} / \partial x_{N}\right)>0\right)$ on the burned gas side of the flame. Accordingly, $T_{2}$ exhibits positive (negative) mean values towards the burned (unburned) gas side of the flame front.

The mean value of the flow stretching term, $T_{3} \times\left(\delta_{t h}^{2} / S_{L}\right)=-\left(S_{i j} n_{j, i}\right)\left(\delta_{t h}^{2} / S_{L}\right)$, has been found to be positive for all cases considered here but the magnitude of the mean value of $T_{3}$ remains small in comparison to that of $T_{2} \times\left(\delta_{t h}^{2} / S_{L}\right)$. Should $\left(x_{T 1}, x_{T 2}\right)$ be the local principal axes of curvature, tangential to a given $c$ isosurface, the flow stretching term can be expressed as $T_{3}=-\left(S_{11} \kappa_{1}+S_{22} \kappa_{2}\right)$, with $S_{11}$ and $S_{22}$ being the tangential strain rates along axes $x_{T 1}$ and $x_{T 2}$, respectively, and $\kappa_{1}$ and $\kappa_{2}$ the principal curvatures. The mean tangential strain rate $a_{T}=S_{11}+S_{22}$ remains positive throughout the flame for this study. A positive value of $T_{3}$ is obtained for a combination of elliptic and hyperbolic scalar topologies [1]. As an example, one obtains $T_{3}>0$ for a combination of $S_{11}>0$ and $S_{22}>0$ for an elliptic concave structure $\left(\kappa_{1}<\right.$ 0 and $\kappa_{2}<0$ ). It has been shown elsewhere [34] that the magnitude of positive mean of normalised tangential strain rate $a_{T} \times \delta_{t h} / S_{L}$ is the highest in case $\mathrm{D}$, with large values of both $u^{\prime} / S_{L}$ and Ka (see Ref. [34] for a physical explanation). The likelihood of obtaining large magnitudes of $S_{11}$ and/or $S_{22}$ and the negative mean curvature of the Bunsen flame (i.e. mean value of $\left(\kappa_{1}+\kappa_{2}\right)$ is negative) lead to the large magnitudes of the mean contribution of $T_{3} \times$ $\left(\delta_{t h}^{2} / S_{L}\right)$ in case $\mathrm{D}$ and it plays a relatively significant role in the curvature budget in this case.

The term $T_{4} \times\left(\delta_{t h}^{2} / S_{L}\right)=-\left\{\left[\left(\partial S_{i j} / \partial x_{i}\right) n_{j}\right] / 2\right\}\left(\delta_{t h}^{2} / S_{L}\right)$, conditional upon $c$, opposes $T_{2} \times$ $\left(\delta_{t h}^{2} / S_{L}\right)$, and they almost balance each other. This means that the two vectors with components 
$n_{j}$ and $\partial S_{i j} / \partial x_{i}$ point in the opposite direction within the flame and towards the burned gas side due to flame normal acceleration, but are directed in the same directions on the unburned side of the flame due to flow divergence ahead of the flame.

The mean contribution to the curvature generation due to vorticity gradients, $T_{5} \times\left(\delta_{t h}^{2} / S_{L}\right)=$ $\left\{\left[\left(\partial W_{i j} / \partial x_{i}\right) n_{j}\right] / 2\right\}\left(\delta_{t h}^{2} / S_{L}\right)$, can alternatively be expressed as $T_{5}=\left\{\left[n_{i} \varepsilon_{i j k}\left(\partial \omega_{k} / \partial x_{j}\right)\right] /\right.$ $4\}\left(\delta_{t h}^{2} / S_{L}\right)$. Using the local principal axes $\left(x_{T 1}, x_{T 2}, x_{N}\right), T_{5}$ can be expressed as $T_{5}=$ $\left(\partial \omega_{2} / \partial x_{1}-\partial \omega_{1} / \partial x_{2}\right) / 4$ for $\boldsymbol{n}=(0,0,1)$, with $\omega_{1}$ and $\omega_{2}$ being the vorticity components along the principal axes of curvature, tangent to the $c$ isosurface. Thus, co-rotating parallel vortices of different intensity and counter-rotating parallel vortices of the same intensity can curve a planar local isosurface element, leading to positive or negative curvatures. For the cases considered here, the mean value of $T_{5}$ remains negligible in comparison to those of $T_{2}$ and $T_{4}$.

\subsection{Flame propagation induced source/sink terms of the curvature transport equation}

The normalised mean values of the various additional terms $\left(T_{6-10} \times \delta_{t h}^{2} / S_{L}\right)$ in eq. (8), conditional upon $c$, for cases A-E are plotted in Figs. 5a-e. The mean value of $\left(\partial S_{d} / \partial x_{N}\right)$ for all cases A-E remains negative for most of the flame front and this quantity correlates weakly with local flame curvature $\kappa_{m}=n_{i, i} / 2$. The predominance of negative values of $\left(\partial S_{d} / \partial x_{N}\right)$ and weak negative $\kappa_{m}$ gives rise to weak positive mean value of $T_{6} \times\left(\delta_{t h}^{2} / S_{L}\right)=$ $\left\{\left(\partial S_{d} / \partial x_{N}\right) n_{i, i} / 2\right\}\left(\delta_{t h}^{2} / S_{L}\right)$. It has been shown [34] that the mean values of $a_{N}$ and $\left(\partial S_{d} / \partial x_{N}\right)$ are almost equal and opposite. Thus, the mean contributions of $T_{1}$ and $T_{6}$ depict opposite trends. 


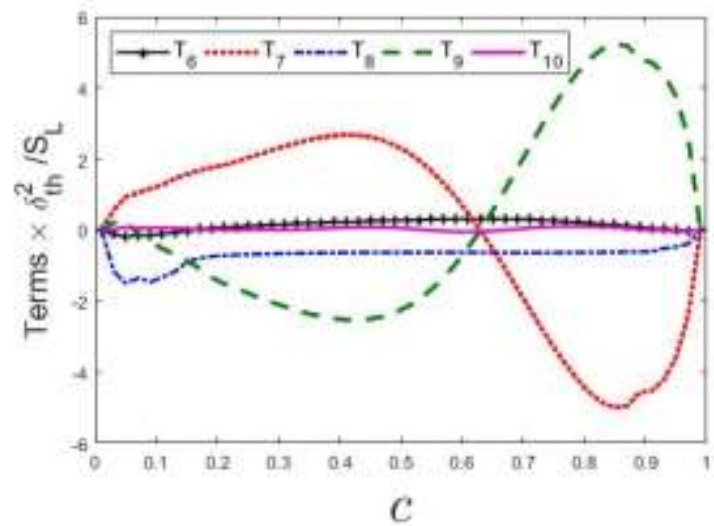

(a)

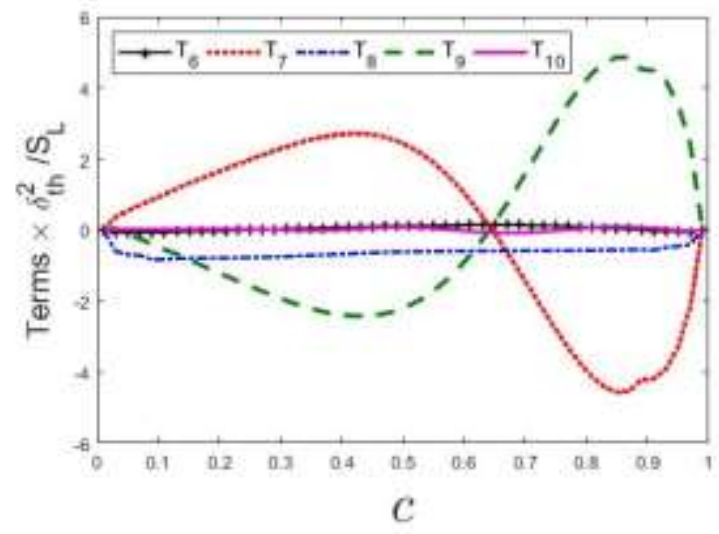

(c)

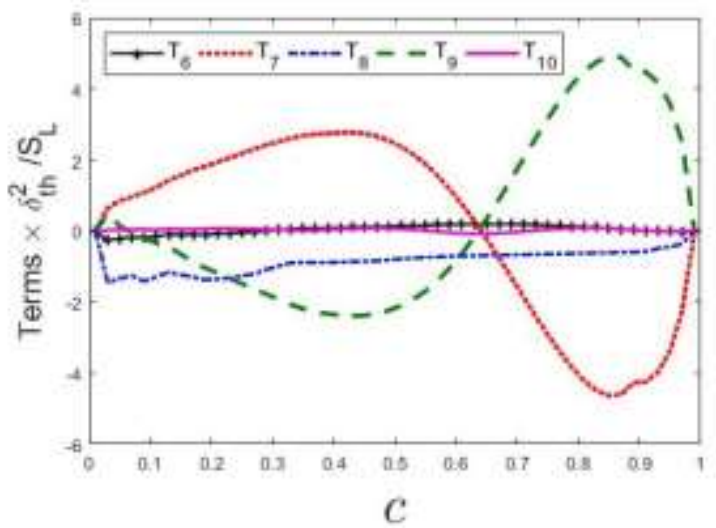

(b)

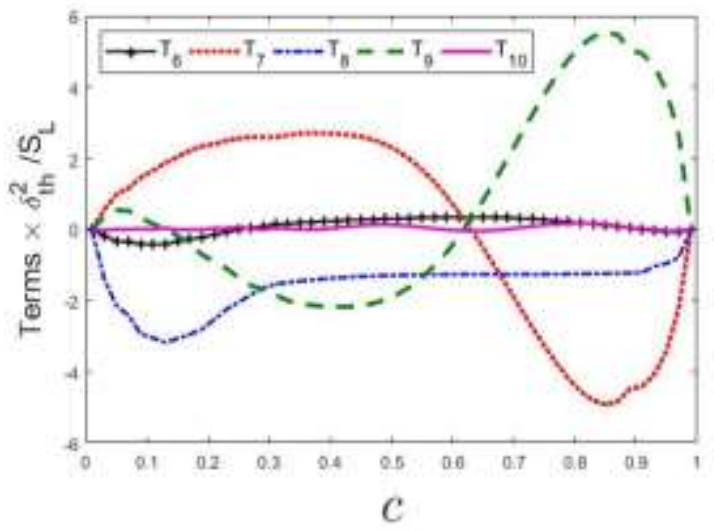

(d)

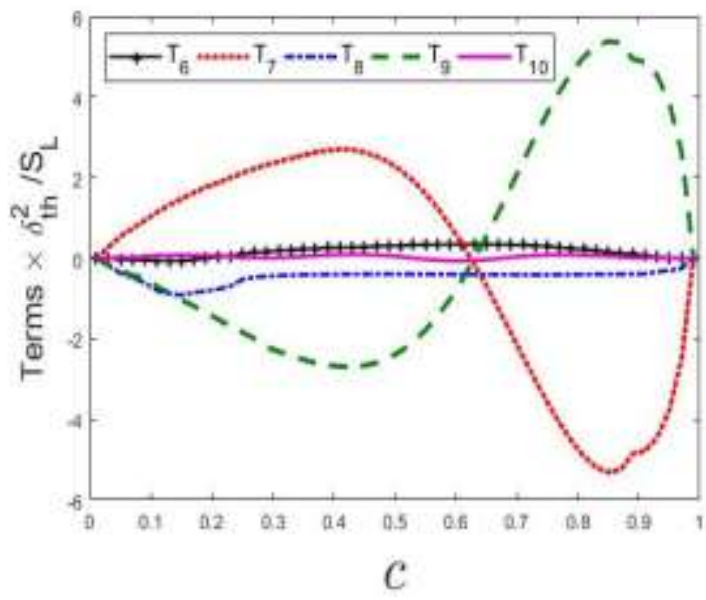

(e)

Figure 5: Profiles of the normalised mean values of the flame propagation induced terms (i.e. $T_{6-10} \times \delta_{t h}^{2} / S_{L}$ ) of the curvature transport equation conditional upon $c$ for cases (a-e) A-E. 
The term $T_{7} \times\left(\delta_{t h}^{2} / S_{L}\right)=\left[\left(\partial^{2} S_{d} / \partial x_{N}^{2}\right) / 2\right]\left(\delta_{t h}^{2} / S_{L}\right)$ originates from the flame normal derivative of the added normal strain rate. The mean variation of $\partial S_{d} / \partial x_{N}$, conditioned upon $c$, has been shown for cases A-E in Ref. [34] and is not repeated here. The mean value of $\partial S_{d} / \partial x_{N}$ decreases from the unburned gas and reaches a minimum before increasing towards the burned gas side. This gives rise to positive mean value of $T_{7}=\left[\left(\partial^{2} S_{d} / \partial x_{N}{ }^{2}\right) / 2\right]$ towards the unburned gas side but the mean contribution of $T_{7}$ becomes negative towards the burned gas side of the flame front.

The mean contribution due to the added stretching term, $T_{8} \times\left(\delta_{t h}^{2} / S_{L}\right)=-\left(S_{i j}^{a} n_{j, i}\right)\left(\delta_{t h}^{2} / S_{L}\right)$, takes negative values throughout the flame for all cases. The magnitude of $T_{8}$ remains smaller than that of $T_{7}$ for flames A-C and E, but the relative contribution of $T_{8}$ is significant in case D. The term $T_{8}$ can be expressed as $T_{8}=-S_{d}\left(\kappa_{1}^{2}+\kappa_{2}^{2}\right)$ using the local principal axes $\left(x_{T 1}, x_{T 2}, x_{N}\right)$ and $\boldsymbol{n}=(0,0,1)$. This explains the predominant negative values of $T_{8}$ in the flame front. Case D represents the thin reaction zones regime of combustion and thus the correlation between $S_{d}$ and curvature, for large values of both $u^{\prime} / S_{L}$ and $K a$, leads to a relatively more important role of $T_{8}$ than in the flames belonging to the wrinkled flamelet and corrugated flamelet regimes (e.g. cases A-C and E).

The second derivatives of $S_{d}$ and of $n_{i}$ and cross products of the gradients of $S_{d}$ and the curvature tensor determine the mean contributions due to $T_{9} \times\left(\delta_{t h}^{2} / S_{L}\right)=-\left\{\left[\left(\partial S_{i j}^{a} / \partial x_{i}\right) n_{j}\right] /\right.$ $2\}\left(\delta_{t h}^{2} / S_{L}\right)$ and $T_{10} \times\left(\delta_{t h}^{2} / S_{L}\right)$. The mean contribution of $T_{9}$ assumes negative (positive) values towards the unburned (burned) gas side of the flame, whereas the magnitude of the mean contribution of $T_{10}$ remains negligible in comparison to that of $T_{9}$. 


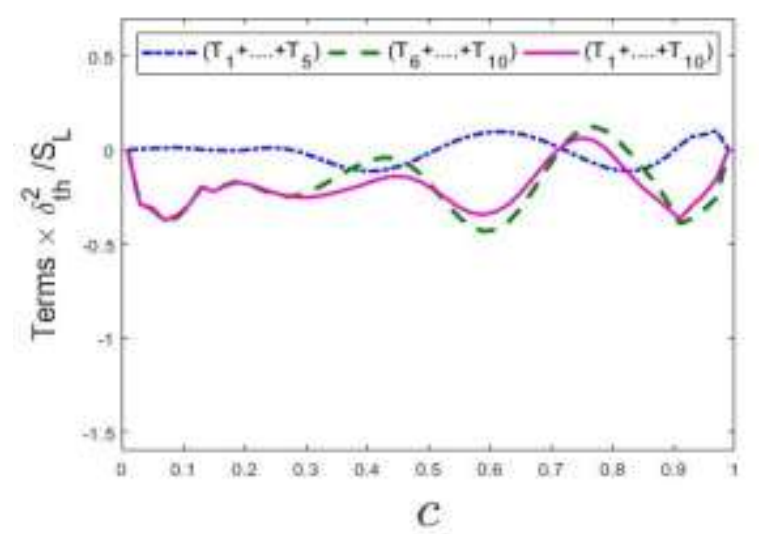

(a)

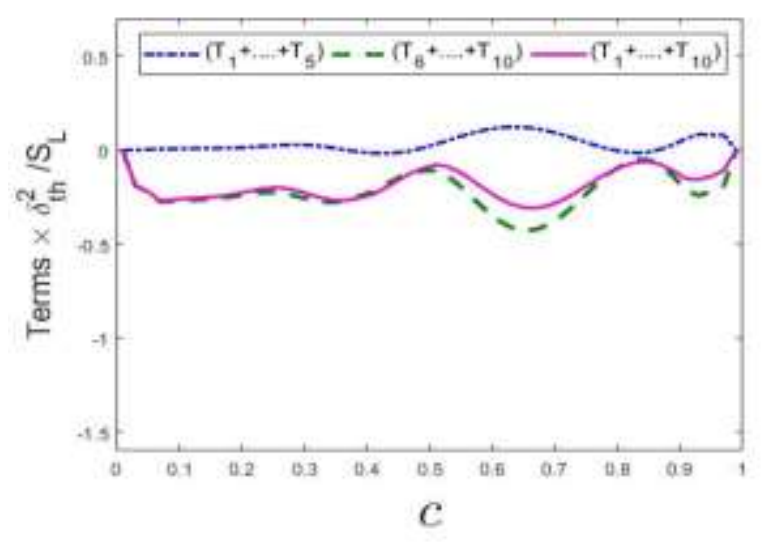

(c)

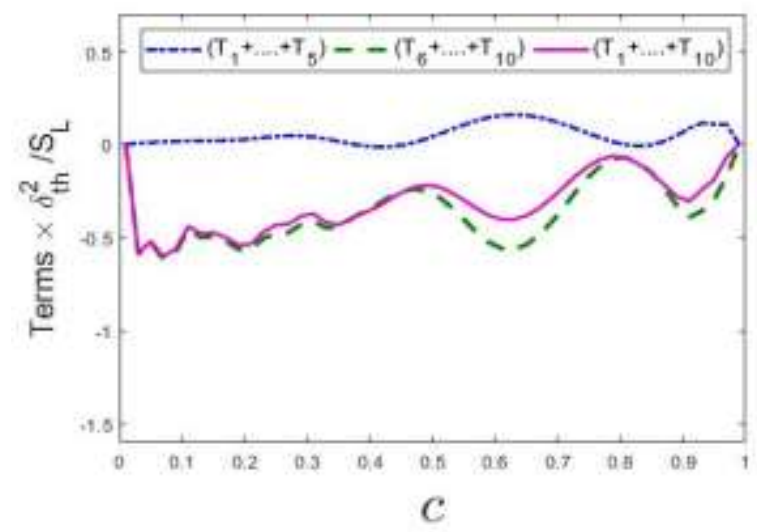

(b)

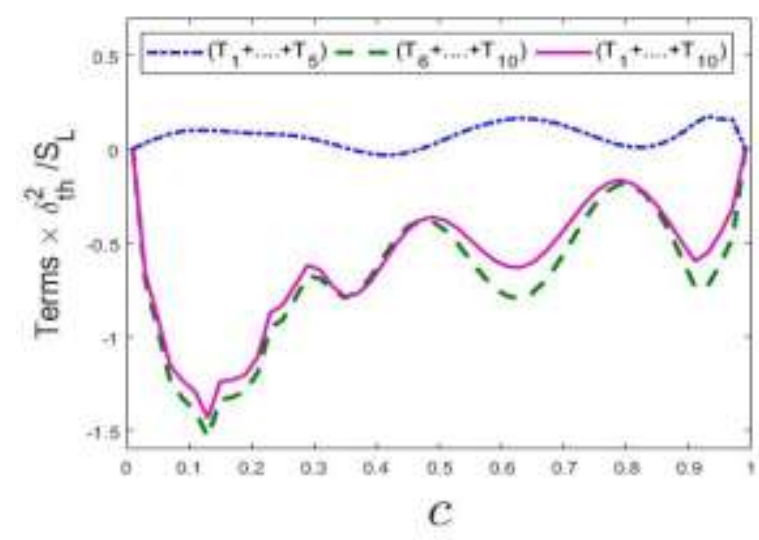

(d)

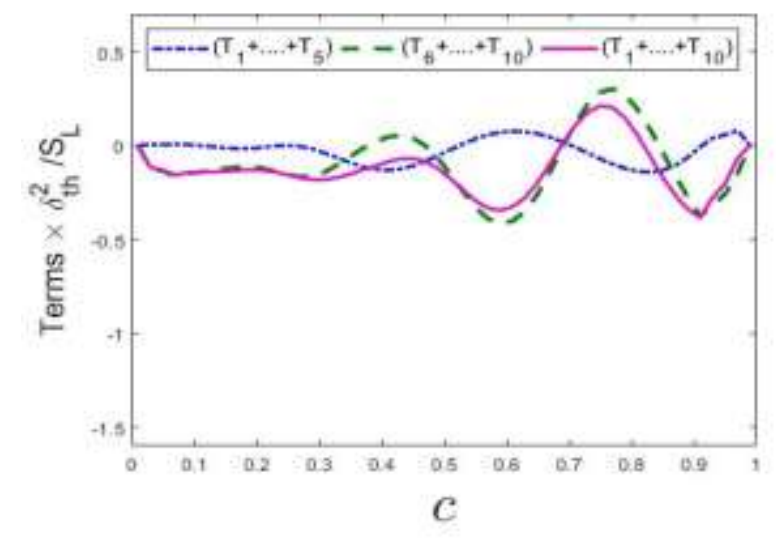

(e)

Figure 6: Profiles of normalised mean values of flow contributions $\left(T_{1}+\cdots+T_{5}\right) \times\left(\delta_{t h}^{2} / S_{L}\right)$, the flame propagation induced contributions $\left(T_{6}+\cdots+T_{10}\right) \times\left(\delta_{t h}^{2} / S_{L}\right)$ and the total contribution $\left(T_{1}+\cdots+T_{10}\right) \times\left(\delta_{t h}^{2} / S_{L}\right)$ to the curvature transport, conditioned on the reaction progress variable $c$ for cases (a-e) A-E. 


\subsection{Overall behaviour of the terms in the curvature transport equation}

Figs. 4 and 5 reveal that the terms $T_{2}, T_{4}, T_{7}, T_{8}$ and $T_{9}$ are the leading order contributors to the curvature budgets for all cases. It is worth noticing that the contributions of $T_{4}$ and $T_{9}$ oppose those of $T_{2}$ and $T_{7}$, respectively. The normalised mean values of flow contributions $\left(T_{1}+\cdots+T_{5}\right) \times\left(\delta_{t h}^{2} / S_{L}\right)$, the added contributions $\left(T_{6}+\cdots+T_{10}\right) \times\left(\delta_{t h}^{2} / S_{L}\right)$ and the total contribution $\left(T_{1}+\cdots+T_{10}\right) \times\left(\delta_{t h}^{2} / S_{L}\right)$ to the curvature evolution, conditioned on the reaction progress variable, $c$, are shown in Fig. 6. It is apparent that the net contribution of the additional terms, due to flame propagation, $\left(T_{6}+\cdots+T_{10}\right) \times\left(\delta_{t h}^{2} / S_{L}\right)$, dominates over the flow sources/sinks, which is consistent with previous findings [41, 42]. The mean contributions of $\left(T_{6}+\cdots+T_{10}\right) \times\left(\delta_{t h}^{2} / S_{L}\right)$ and $\left(T_{1}+\cdots+T_{10}\right) \times\left(\delta_{t h}^{2} / S_{L}\right)$ remain negative and close to each other throughout the flame front for the elevated pressure cases (B and C) and also for the atmospheric pressure flame $\mathrm{D}$, which represents the thin reaction zones regime; for flames BD the net flow contribution $\left(T_{1}+\cdots+T_{5}\right) \times\left(\delta_{t h}^{2} / S_{L}\right)$ remains mostly positive.. However, for the atmospheric cases $\mathrm{A}$ and $\mathrm{E}$, the mean value of $\left(T_{6}+\cdots+T_{10}\right) \times\left(\delta_{t h}^{2} / S_{L}\right)$ assumes positive values in some regions within the flame, in spite of exhibiting mostly negative values in the major part of the flame front.

\subsection{Relations of the terms of the curvature transport equation with local curvature}

The mean flow $\left(T_{1-5}\right)$ and added contributions $\left(T_{6-10}\right)$ to the curvature transport, conditioned upon the normalised curvature $\kappa_{m} \delta_{t h}$, are shown for $c=0.8$ isosurface in Figs. 7 and 8 , respectively. 


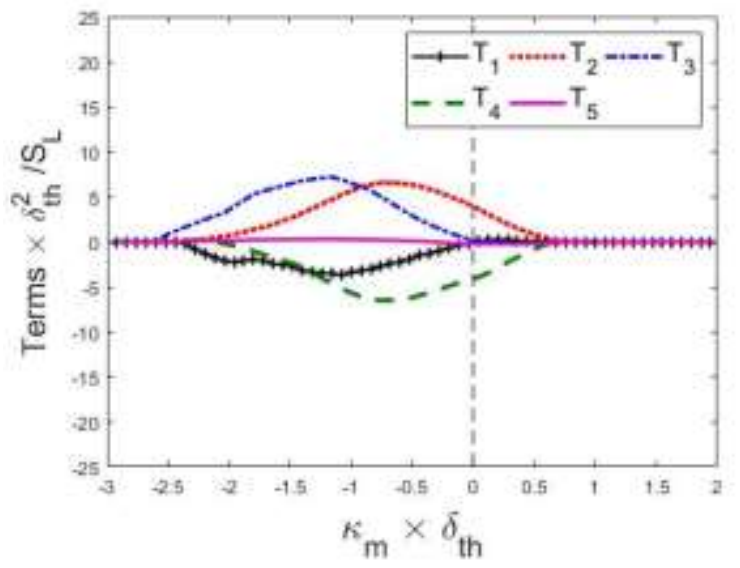

(a)

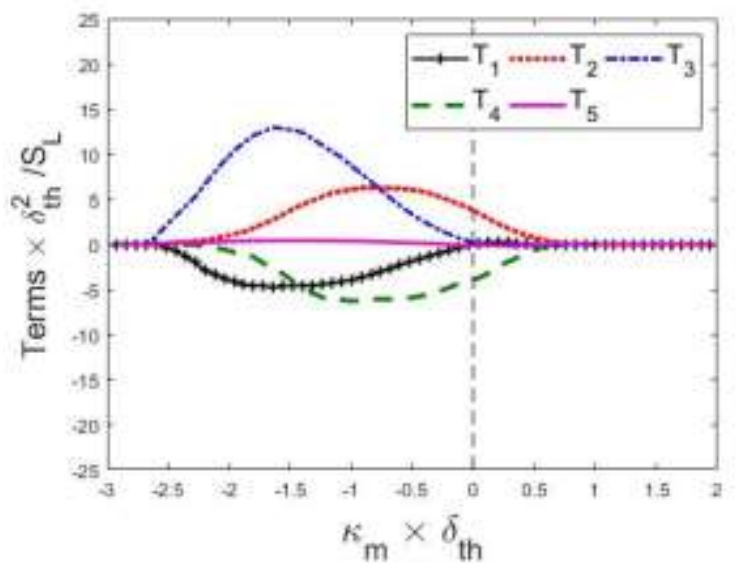

(c)

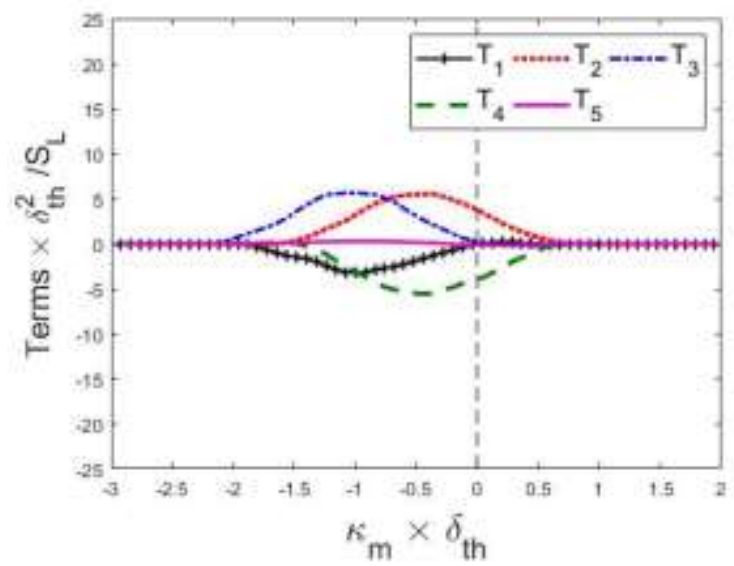

(b)

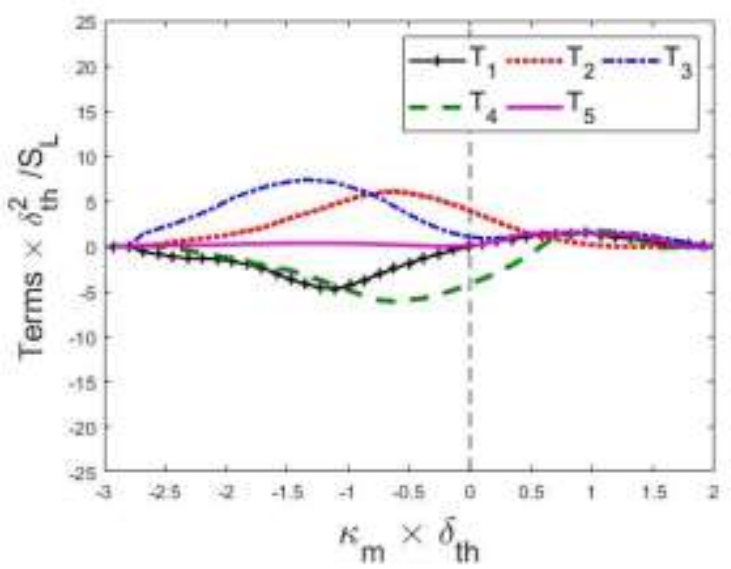

(d)

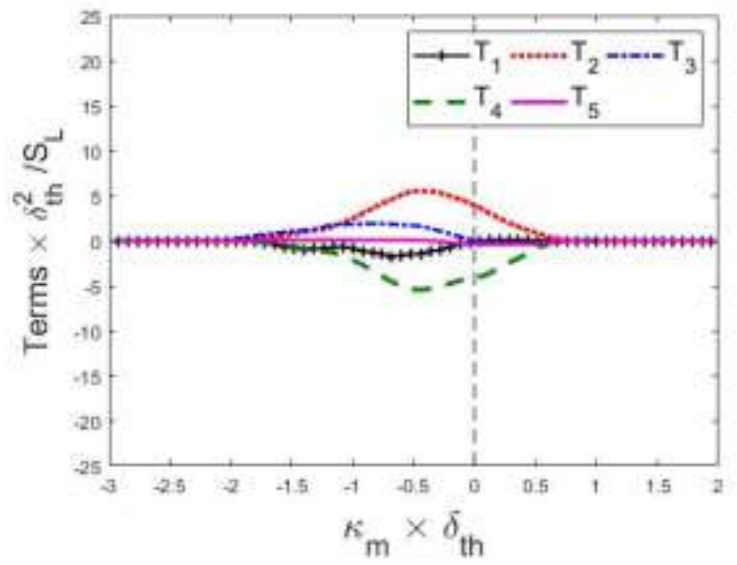

(e)

Figure 7: Profiles of the normalised mean values of the fluid flow induced terms (i.e. $T_{1-5} \times$ $\delta_{t h}^{2} / S_{L}$ ) of the curvature transport equation conditional upon normalised curvature $\kappa_{m} \times \delta_{t h}$ on $c=0.8$ isosurface for cases (a-e) A-E. 


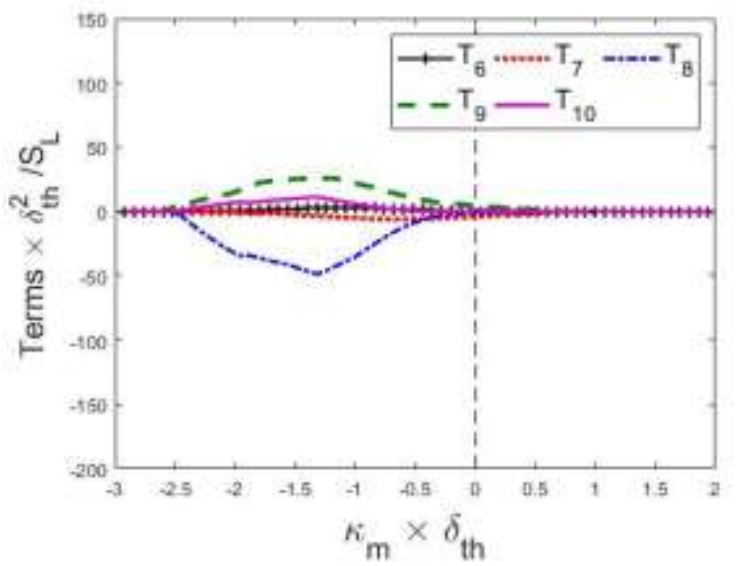

(a)

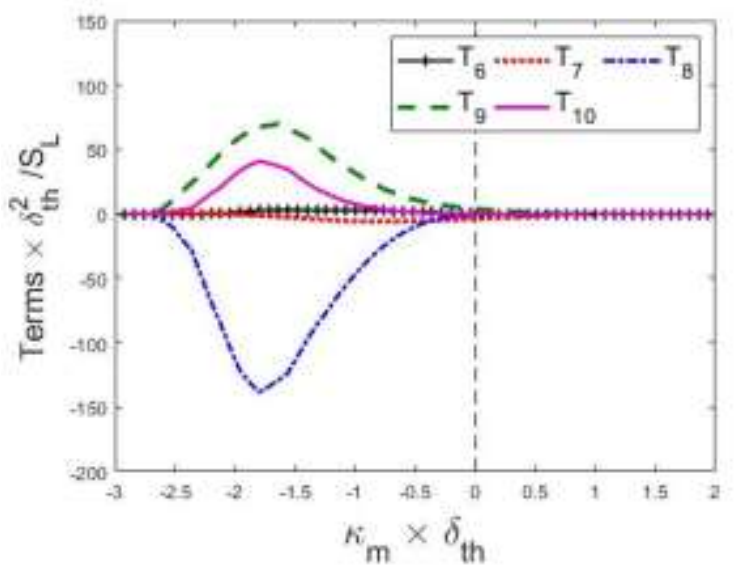

(c)

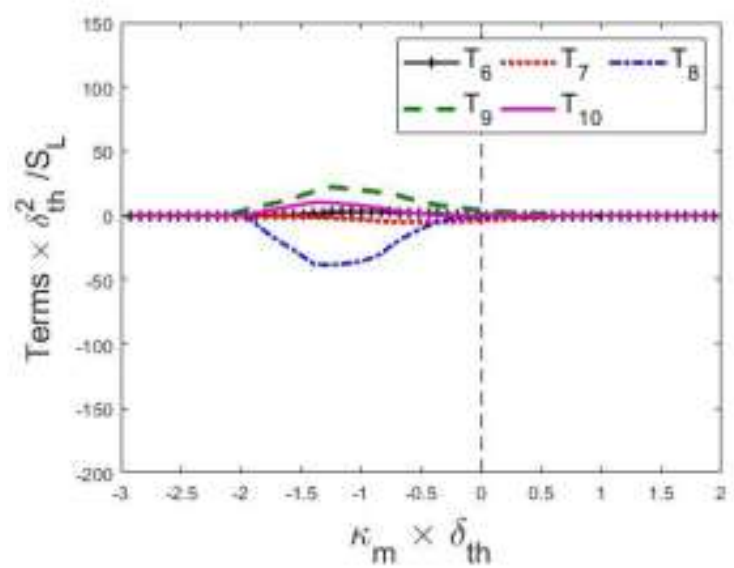

(b)

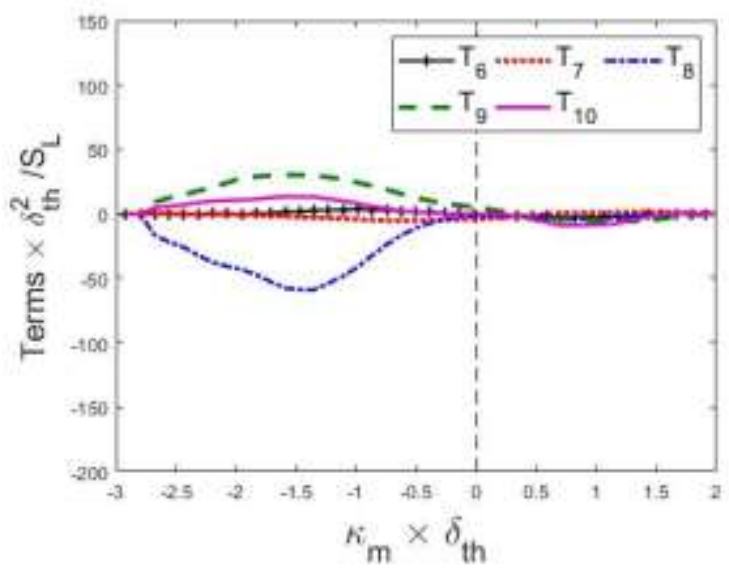

(d)

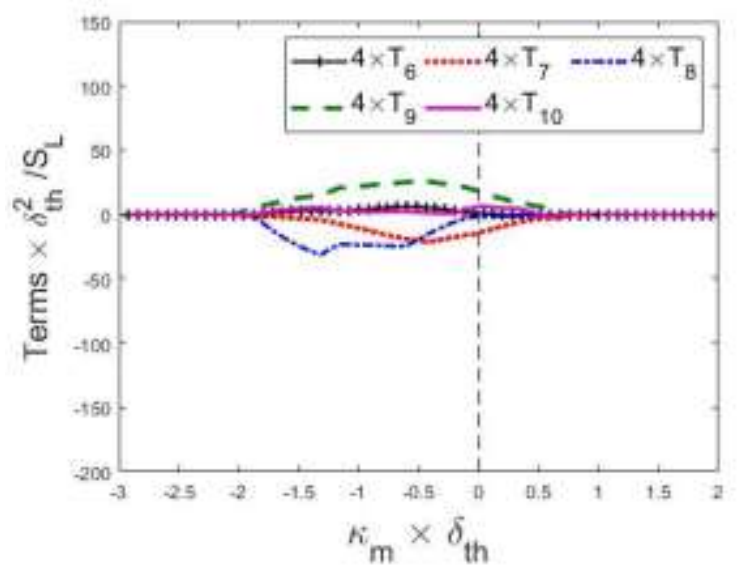

(e)

Figure 8: Profiles of the normalised mean values of the flame propagation induced terms (i.e. $T_{6-10} \times \delta_{t h}^{2} / S_{L}$ ) of the curvature transport equation conditional upon normalised curvature $\kappa_{m} \times \delta_{t h}$ on $c=0.8$ isosurface for cases (a-e) A-E. 
It is worth noting that the $c=0.8$ isosurface is chosen because the reaction rate $\dot{w}_{c}$ attains its maximum value close to this value of $c$, and thus the $c=0.8$ isosurface can be considered as representative of the flame surface for this analysis following several previous studies [17$21,23,24,28]$. The mean values of all the terms conditional on $\kappa_{m}$ vanish for both large positive and negative magnitudes of $\kappa_{m}$. As a rule, in Figs. 7 and 8 the positive contributions of the terms in the positively curved patches tend to increase the convexity of the flame, whereas the negative contributions of the terms act to reduce the convexity at $\kappa_{m}>0$. By the same token, the positive contributions of the terms in the negatively curved surface elements tend to decrease the concavity of the flame, whereas negative contributions act to increase the concavity at $\kappa_{m}<0$.

The mean values of $T_{1}=\left[\left(a_{N} n_{i, i}\right) / 2\right]$ in Fig. 7 shows mostly negative value for negative curvatures, but a small increasing trend with an increase in $\kappa_{m}$ is observed for all cases considered here. As $a_{N}$ is predominantly positive, the variation of $T_{1}$ is determined by curvature $\kappa_{m}=n_{i, i} / 2$ because the correlation between $a_{N}$ and $\kappa_{m}$ remains weak in these flames [34]. The mean value of $T_{2}$ conditional on curvature exhibits positive values for cases A-E, which is consistent with positive conditional mean values of $T_{2}$ for $c=0.8$ in Fig. 4 . The mean values of the flow stretching term $T_{3}=-\left(S_{i j} n_{j, i}\right)$, conditional on curvature $\kappa_{m}$, exhibits positive values, with maxima at highly negative curvatures, in all cases A-E, and decreases with increasing curvature for $\kappa_{m} \delta_{t h} \geq-1.5$. The mean value of $T_{4}$, conditioned on curvature, assumes negative values, which is consistent with negative conditional mean values of $T_{4}$ for $c=0.8$ in Fig. 4 . The mean value of $T_{5}$ conditioned on curvature remains negligible for all cases. 
The mean contribution of the additional normal strain rate term, $T_{6}=\left\{\left(\partial S_{d} / \partial x_{N}\right) n_{i, i} / 2\right\}$, conditioned on curvature, assumes negligible values. The mean value of the term $T_{7}$ due to flame normal gradient of the added normal strain rate, conditioned on curvature, is also negligible in comparison to the leading order contributions of $T_{8}$ and $T_{9}$ for all flames. The mean contribution due to the added stretching term, $T_{8}=-\left(S_{i j}^{a} n_{j, i}\right)$, remains negative for all cases. It has already been discussed that $T_{8}$ can be approximated as $T_{8}=-S_{d}\left(\kappa_{1}^{2}+\kappa_{2}^{2}\right)$ in the coordinates aligned with principal axes of curvature, which suggests a cubic curvature dependence of $T_{8}$ due to curvature dependence of displacement speed $S_{d}$ (i.e., a negative correlation between $S_{d}$ and $\kappa_{m}$ in these cases) [11-21,34]. The mean contribution of the term $T_{9}$ due to the added strain rate gradients, conditional upon curvature $\kappa_{m}$, assume large positive values in all cases considered here. The mean value of the added vorticity curl contribution, $T_{10}$, conditional upon $\kappa_{m}$ remains negligible in all these cases.

The normalised mean values of flow contributions $\left(T_{1}+\cdots+T_{5}\right) \times\left(\delta_{t h}^{2} / S_{L}\right)$, the flame propagation induced contributions $\left(T_{6}+\cdots+T_{10}\right) \times\left(\delta_{t h}^{2} / S_{L}\right)$ and the total contribution $\left(T_{1}+\cdots+T_{10}\right) \times\left(\delta_{t h}^{2} / S_{L}\right)$ to the curvature transport, conditioned on the curvature $\kappa_{m}$ value, on the $c=0.8$ isosurface are shown in Figs. 9a-e for cases A-E respectively. It can be seen from Fig. 9 that the magnitude of the mean value of the total flow contributions $\left(T_{1}+\cdots+T_{5}\right)$ remains smaller than that of the average additional terms, induced by the flame propagation, $\left(T_{6}+\cdots+T_{10}\right)$, in all cases. This is consistent with the observations made previously on Fig. 6 and with previous findings $[41,42]$. It can further be seen from Figs. 9a-e that the net mean contributions of $\left(T_{6}+\cdots+T_{10}\right)$ and $\left(T_{1}+\cdots+T_{10}\right)$ assume negative values for highly negative curved elements. However, the large negative mean contributions of $\left(T_{6}+\cdots+T_{10}\right)$ and $\left(T_{1}+\cdots+T_{10}\right)$ for flame $\mathrm{D}$ at highly curved convex patches are much greater in magnitude than those observed for highly concave elements. The opposite is apparent in cases A-C and E. 


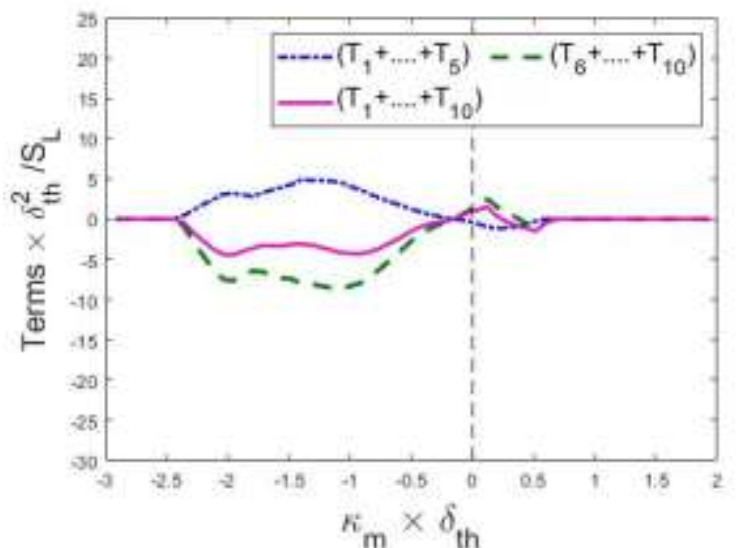

(a)

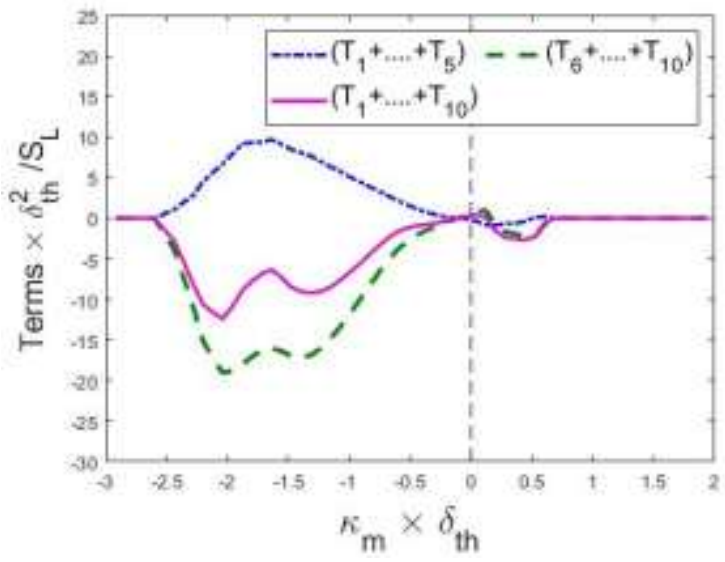

(c)

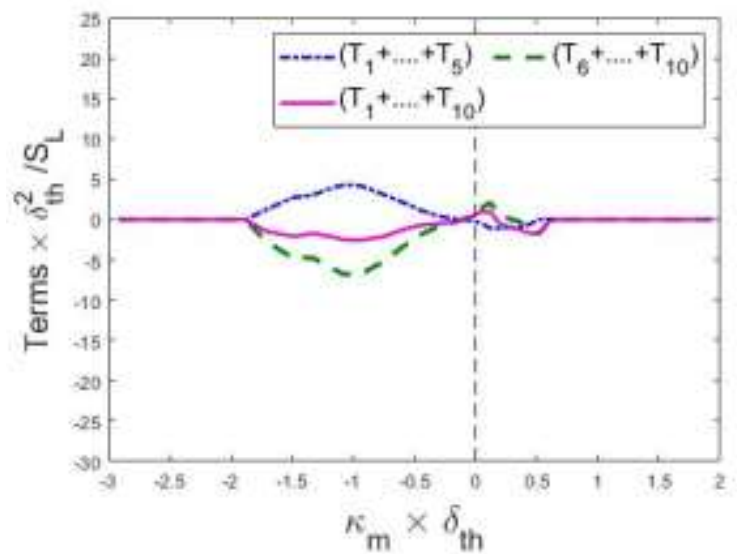

(b)

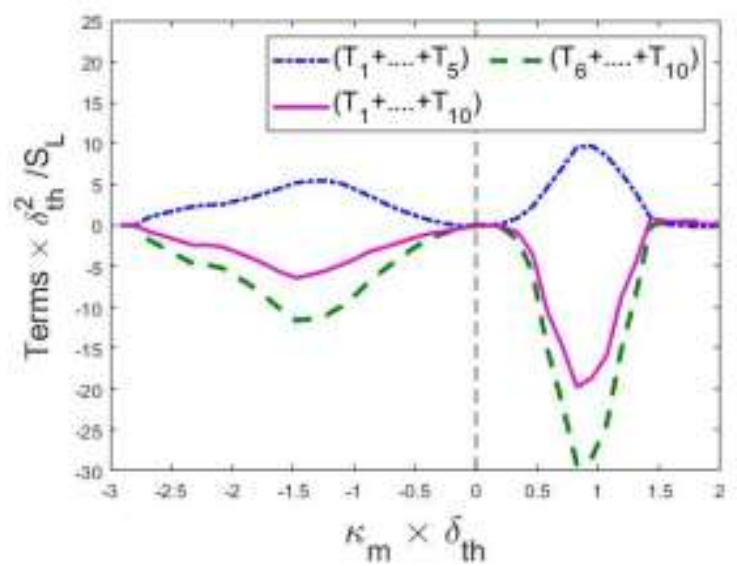

(d)

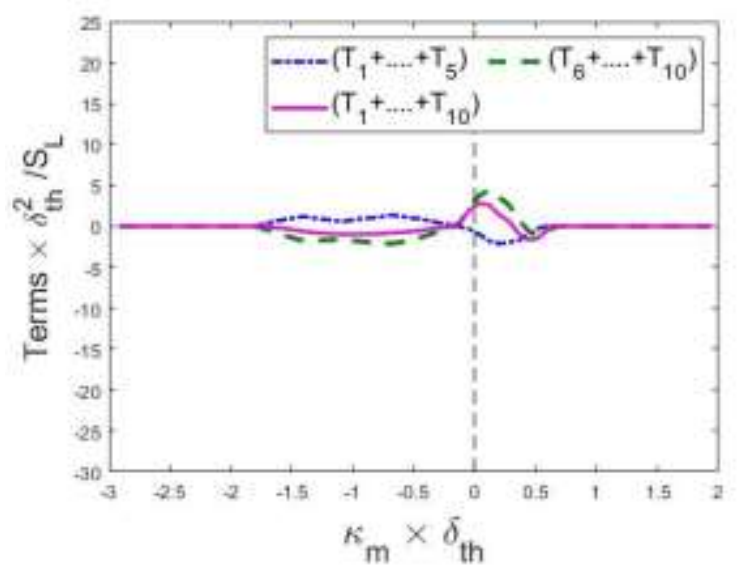

(e)

Figure 9: Profiles of normalised mean values of flow contributions $\left(T_{1}+\cdots+T_{5}\right) \times\left(\delta_{t h}^{2} / S_{L}\right)$, the flame propagation induced contributions $\left(T_{6}+\cdots+T_{10}\right) \times\left(\delta_{t h}^{2} / S_{L}\right)$ and the total contribution $\left(T_{1}+\cdots+T_{10}\right) \times\left(\delta_{t h}^{2} / S_{L}\right)$ to the curvature transport conditional upon normalised curvature $\kappa_{m} \times \delta_{t h}$ on $c=0.8$ isosurface for cases (a-e) A-E. 
Moreover, the magnitude of negative mean contributions of $\left(T_{6}+\cdots+T_{10}\right)$ and $\left(T_{1}+\cdots+\right.$ $T_{10}$ ) at the negatively curved location is the highest in case C. This suggests that negatively curved zones are more likely to occur in Bunsen flames because of their inherent configuration (i.e. where flame has a negative mean curvature determined by the flow configuration) and they are promoted because of negative net mean contribution of $\left(T_{1}+\cdots+T_{10}\right)$. Furthermore, flame propagation effects act to reduce the convexity at the positively curved bulges in these flames in a mean sense but this does not preclude the occurrences of locally positively curved bulges because of the prevailing conditions in terms of local flame-turbulence interaction. Figure 9 indicates that sharply curved locations with large negative curvatures and moderately positively curved bulges are likely to be obtained in case $\mathrm{C}$, which is consistent with the presence of the D-L instability in this flame. This also leads to negative skewness of PDF of mean curvature in the cases where the effects of the D-L instability are strong [39]. The curvature dependence of displacement speed $S_{d}$ is expected to be stronger in case D than in cases A-C and $\mathrm{E}$ because case D belongs to the thin reaction zones regime of combustion [44]. Thus, the local displacement speed can assume locally negative values in highly positive curved locations [11-21,34] in case D and this severely limits the possibility of forming wrinkles with large positive curvature magnitudes. This is reflected in the high negative mean contributions of $\left(T_{6}+\cdots+T_{10}\right)$ and $\left(T_{1}+\cdots+T_{10}\right)$ at the positively curved locations in case D.

The mean values of $T_{1-5} \times\left(\delta_{t h}^{2} / S_{L}\right)$ conditional on $\kappa_{m} \times \delta_{t h}$ and $\kappa_{g} \times \delta_{t h}^{2}$ for cases A, C, D and $\mathrm{E}$ are shown Fig. 10 for $c=0.8$ isosurface. The corresponding variations of $T_{6-10} \times$ $\left(\delta_{t h}^{2} / S_{L}\right)$ conditional on $\kappa_{m} \times \delta_{t h}$ and $\kappa_{g} \times \delta_{t h}^{2}$ for these cases for the $c=0.8$ isosurface are shown in Fig. 11. Case B is not shown in Figs. 10 and 11 for its qualitative similarity with case C. 

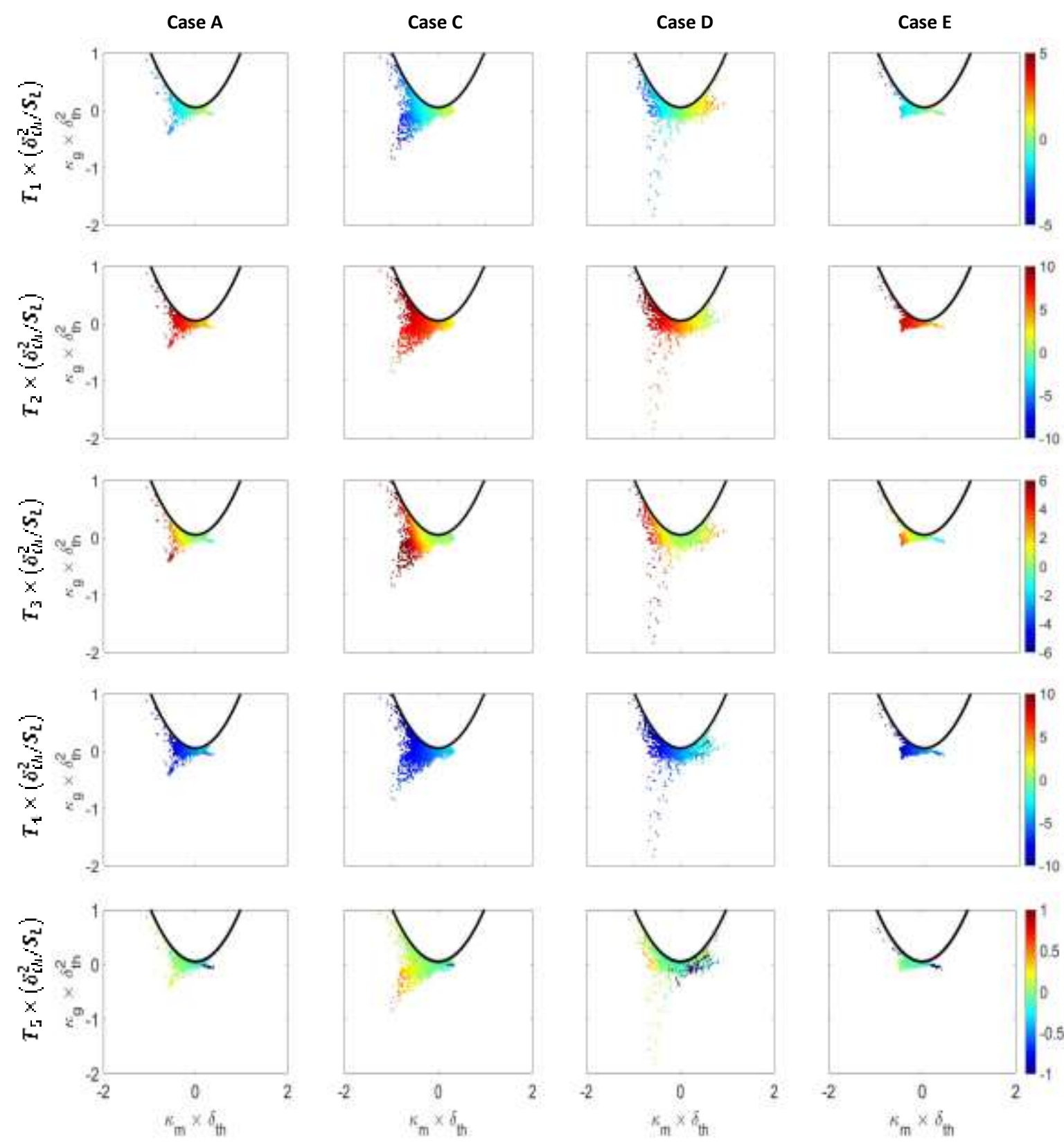

Figure 10: Variations of the mean values of $T_{1-5} \times\left(\delta_{t h}^{2} / S_{L}\right)\left(1^{\text {st }}-5^{\text {th }}\right.$ row $)$ conditional on $\kappa_{m} \times$ $\delta_{t h}$ and $\kappa_{g} \times \delta_{t h}^{2}$ for cases A, C, D and E ( $1^{\text {st }}-4^{\text {th }}$ column $)$ for $c=0.8$ isosurface. 

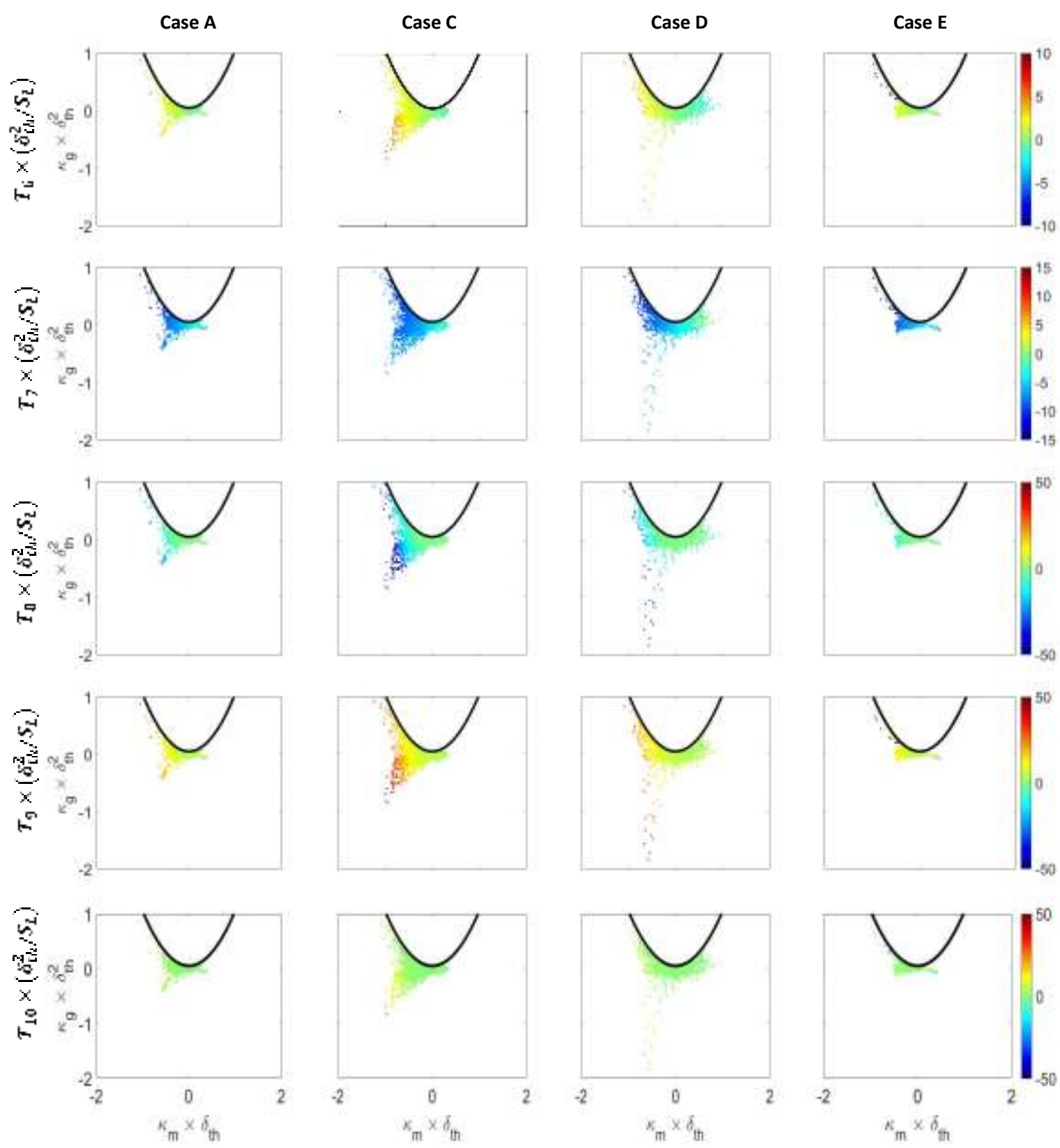

Figure 11: Variations of the mean values of $T_{6-10} \times\left(\delta_{t h}^{2} / S_{L}\right)\left(1^{\text {st }}-5^{\text {th }}\right.$ row $)$ conditional on $\kappa_{m} \times$ $\delta_{t h}$ and $\kappa_{g} \times \delta_{t h}^{2}$ for cases A, C, D and E ( $1^{\text {st }}-4^{\text {th }}$ column $)$ for $c=0.8$ isosurface. 
It is evident from Figs. 10-11 that the distribution is mostly concentrated for negative values of $\kappa_{m}$. Figures 10-11 show that the high magnitudes of the mean values of $T_{1-10}$ are obtained for negative curvature values especially for saddle concave (i.e. $\kappa_{m}<0$ and $\kappa_{g}<0$ ) and tile concave (i.e. $\kappa_{m}<0$ and $\kappa_{g}=0$ ) flame topologies. The large magnitudes of $T_{1-10} \times\left(\delta_{t h}^{2} / S_{L}\right)$ for negative curvature locations are consistent with the findings in Figs. 7 and 8. However, the tendency of obtaining large magnitudes of the mean values of $T_{1-10}$ for saddle concave (i.e. $\kappa_{m}<0$ and $\left.\kappa_{g}<0\right)$ flame topologies is particularly strong for case C. The probability of finding negative principal curvature increases in case $\mathrm{C}$ due to the formation of cusps as a result of the D-L instability and this is evident from the curvature transport statistics in this case. It is worth noting (see Figs. 1 and 3) that a moderate amount of cusp formation takes place due to Huygens propagation in case A [39] but Figs. 7-11 indicate that the curvature transport statistics for cusp formation in case $\mathrm{C}$ due to the $\mathrm{D}$-L instability is fundamentally different from that in case of Huygens propagation.

The case D represents the thin reaction zones regime combustion and has the highest value of $u^{\prime} / S_{L}$ and thus the extent of flame wrinkling in this case is greater than in cases A and E. This gives rise to higher probability of obtaining negative principal curvatures and saddle point flame topologies in case D than in cases A and E. Moreover, the greater extent of flame wrinkling in case $\mathrm{D}$ also leads to non-negligible magnitudes of $T_{1-10} \times\left(\delta_{t h}^{2} / S_{L}\right)$ for larger range of positive $\kappa_{m}$ values than in cases $\mathrm{A}$ and $\mathrm{E}$. The relative distributions of $T_{1-10} \times$ $\left(\delta_{t h}^{2} / S_{L}\right)$ in relation to $\kappa_{m}$ have been found to be consistent with the variations of these terms conditional on $\kappa_{m}$ in Figs. 7 and 8, and thus the corresponding description is not repeated here for the sake of brevity. 
The above findings indicate that the added contributions to the curvature $\kappa_{m}$ transport arising from displacement speed $S_{d}$ play significant roles for all flames considered here. This essentially indicates that curvature and displacement speed are intrinsically interlinked. In other words, displacement speed $S_{d}$ is not only interlinked with curvature (i.e. a negative correlation exists between $S_{d}$ and $\kappa_{m}$ [9-21,23-25,27,28]) but it also affects flame wrinkling through the curvature evolution. It has been demonstrated elsewhere [60-63] that the curvature $\kappa_{m}$ and its interrelation with displacement speed $S_{d}$ play a key role in the Flame Surface Density (FSD) and scalar dissipation rate (SDR) transports. Furthermore, the analysis of curvature evolution also reveals that the displacement speed induced terms are principally responsible for the generation of negatively curved cusps in the case of the D-L instabilities in high-pressure flames.

\section{CONCLUSIONS}

The evolution of flame curvature in turbulent premixed Bunsen flames at three different values of thermodynamic pressure has been analysed in detail. In order to distinguish the pressure effects, two additional cases at the lowest pressure value have been considered by changing inlet turbulence intensity $u^{\prime} / S_{L}$ and integral length scale to flame thickness ratio, $l / \delta_{t h}$, respectively, in such a manner that the turbulent Reynolds number, $R e_{t}$, remains identical to the highest-pressure case. It has been found that all Bunsen flame cases show predominant probability of finding negative curvature and concave cup flame topology. However, the probability of finding concave saddle point flame topology, typical of cusp formation, increases with increasing pressure and this is particularly prominent for the case with the highest pressure due to the presence of the D-L instability. The probability of finding positive curvature and convex saddle point flame topologies also increases with increasing $u^{\prime} / S_{L}$. The net mean contribution of the terms arising from flame propagation in the flame curvature transport 
equation (i.e., $\left.\left(T_{6}+\cdots+T_{10}\right)\right)$ dominates the net mean contribution of the flow terms (i.e., $\left.\left(T_{1}+\cdots+T_{5}\right)\right)$. The net mean contribution of $\left(T_{1}+\cdots+T_{10}\right)$ remains negative for both negatively and positively curved regions for all cases on the most reactive $c$-isosurface, apart from localised positive peaks for small positive curvature values found in some cases (e.g. A, B and E). By contrast, the net mean contribution of $\left(T_{1}+\cdots+T_{10}\right)$ assumes high negative values at the negatively curved regions for high-pressure flames, which in turn is responsible for negative skewness in the curvature PDF for these cases where the effects of the D-L instability are prominent. This indicates that the contributions arising from the displacement speed are principally responsible for these negatively curved cusp formations in the case of DL instability. It has been found that large magnitudes of the terms of the curvature transport equation (i.e. $T_{1-10}$ ) are obtained also for saddle point type (i.e., $\kappa_{g}<0$ ) flow topologies. The present study reveals that the flame topology distribution and the curvature evolution are likely to be different for low-pressure flames in comparison to high-pressure conditions for the same turbulence parameters. However, an increase in $u^{\prime} / S_{L}$ in low-pressure condition alters the distributions of curvature and flame topologies.

\section{ETHICS STATEMENT}

This work did not involve any active collection of human data

\section{COMPETING INTERESTS STATEMENT}

We have no competing interests.

\section{FUNDING}

The authors are grateful to EPSRC, UK (EP/R029369/1, EP/P022286/1), the German Research Foundation (DFG, KL1456/5-1) ARCHER, Rocket HPC and Gauss Centre for Supercomputing (grant: pn69ga) for the financial and computational supports. 


\section{REFERENCES}

1. Dopazo, C., Martin, J. and Hierro, J.: Local geometry of isoscalar surfaces, J. Phys. Rev. E, 76, $056316(2007)$

2. Cant, R.S., Rutland, C.J., and Trouvé, A.: Statistics for laminar flamelet modelling, Proc. Summer Prog. 1990, Center for Turbulence Research, Stanford, pp. 271-279 (1990)

3. Poinsot, T. and Veynante, D.: Theoretical and numerical combustion, R.T.Edwards Inc., Philadelphia, USA (2001)

4. Pelcé, P.: (Ed.), Dynamics of Curved Fronts, Academic Press Inc. (1988)

5. Markstein, G.H.: Experimental and Theoretical Studies of Flame-Front Stability, J. Aero. Sci., 18, 199-209 (1951)

6. Clavin, P. and Joulin, G.: Premixed flames in large scale and high intensity turbulent flows, J. Phys. Lett. 44, L-1-L-12 (1983)

7. Mikolaitis, D.W.: The interaction of flame curvature and stretch, part 1: The concave premixed flame, Combust. Flame, 57, 25-31 ( 1984)

8. Mikolaitis, D.W.: The interaction of flame curvature and stretch, part 2: The convex premixed flame, Combust. Flame 58, 23-29 (1984)

9. Echekki, T., and Chen, J.H.: Unsteady strain rate and curvature effects in turbulent premixed methane-air flames, Combust. Flame, 106, 184-202 (1996)

10. Renou. B., Boukhalfa, A., Peuchberty, D., and Trinité, M.: Effects of stretch on the local structure of freely propagating premixed low-turbulent flames with various Lewis numbers, Proc. Combust. Inst., 29,841-847 (1998)

11.Peters, N., Terhoeven, P., Chen, J.H., and Echekki, T.: Statistics of Flame Displacement Speeds from Computations of 2-D Unsteady Methane-Air Flames”, Proc. Combust. Inst., 27,833-839 (1998) 
12.Echekki, T. and Chen, J.H.: Analysis of the contributions of curvature to premixed flame propagation, Combust. Flame, 118, 308-311 (1999)

13.Chen, J., H., and Im, H.G.: Correlation of flame speed with stretch in turbulent premixed Methane/Air flames, Proc. Combust. Inst., Pittsburgh, 27, 819-826 (1998)

14.Chen, J., H., and Im, H.G.: Stretch effects on the burning velocity of turbulent premixed Hydrogen/Air flames, Proc. Combust. Inst., 28, 211-218 (2000)

15.Hawkes, E.R., and Chen, J.H.: Direct numerical simulation of hydrogen-enriched lean premixed methane air flames. Combust. Flame, 138, 242-258 (2004)

16.Hawkes, E.R., and Chen, J.H.: Evaluation of models for flame stretch due to curvature in the thin reaction zones regime, Proc. Combust. Inst., 30, 647-655 (2005)

17.Chakraborty, N. and Cant, S.: Unsteady effects of strain rate and curvature on turbulent premixed flames in inlet-outlet configuration, Combust. Flame 137, 129-147 (2004)

18.Chakraborty, N. and Cant, R.S.: Influence of Lewis number on curvature effects in turbulent premixed flame propagation in the thin reaction zones regime, Phys. Fluids, 17, 105105 (2005)

19.Chakraborty, N. and Cant, R.S.: Effects of strain rate and curvature on surface density function transport in turbulent premixed flames in the thin reaction zones regime, Phys. Fluids, 17, 65108 (2005)

20.Jenkins, K.W., Klein, M., Chakraborty, N. and Cant, R.S.: Effects of strain rate and curvature on the propagation of a spherical flame kernel in the thin reaction zones regime, Combust. Flame, 145, 415-434 (2006)

21.Klein, M., Chakraborty, N., Jenkins, K.W., Cant, R.S.: Effects of initial radius on the propagation of spherical premixed flame kernels in turbulent environment, Phys. Fluids, 18, $055102(2006)$ 
22.Savarianandam VR and Lawn C.J.: Burning velocity of premixed turbulent flames in the weakly wrinkled regime, Combust Flame, 146, 1-18 (2006)

23. Chakraborty, N.: Comparison of displacement speed statistics of turbulent premixed flames in the regimes representing combustion in corrugated flamelets and thin reaction zones, Phys. Fluids, 19,105109 (2007)

24.Chakraborty, N., Hawkes, E.R., Chen, J.H. and Cant, R.S.: Effects of strain rate and curvature on Surface Density Function transport in turbulent premixed CH4-air and H2-air flames: A comparative study, Combust. Flame, 154, 259-280 (2008)

25.Han, I., Huh, K.Y.: Roles of displacement speed on evolution of flame surface density for different turbulent intensities and Lewis numbers in turbulent premixed combustion, Combust. Flame, 152, 194-205 (2008)

26.Hartung, G., Hult, J., Balachandran, R., Mackley, M.R. and Kaminski, C.F.: Flame front tracking in turbulent lean premixed flames, Applied Physics B, 96, 843-862 (2009)

27.Chakraborty, N., Klein, M., Cant, R.S.: Effects of turbulent Reynolds number on the displacement speed statistics in the thin reaction zones regime turbulent premixed combustion, J. Combust., 473679 (2011)

28.Chakraborty, N., Hartung, G., Katragadda, M., Kaminski, C. F.: A numerical comparison of 2D and 3D density-weighted displacement speed statistics and implications for laser based measurements of flame displacement speed, Combust. Flame, 158, 1372-1390 (2011)

29.Kerl, J., Lawn, C. and Beyrau, F.: Three-dimensional flame displacement speed and flame front curvature measurements using quad-plane PIV, Combust. Flame, 160, 2757-2769 (2013)

30.Giannakopoulos, G.K., Matalon, M., Frouzakis, C.E. and Tamboulides, A.G.: The curvature Markstein length and the definition of flame displacement speed for stationary spherical flames, Proc. Combust. Inst., 35, 737-743 (2015) 
31.Chakraborty, N. and Klein, M.: Influence of Lewis number on the Surface Density Function transport in the thin reaction zones regime for turbulent premixed flames, Phys. Fluids, 20, $065102(2008)$

32.Chakraborty, N. and Klein, M.: Effects of global flame curvature on surface density function transport in turbulent premixed flame kernels in the thin reaction zone regime, Proc. Combust. Inst., 32, 1435-1443 (2009)

33.Sandeep, A., Proch, F., Kempf, A.M., Chakraborty, N.: Statistics of strain rates and Surface Density Function in a flame-resolved high-fidelity simulation of a turbulent premixed bluff body burner, Phys. Fluids, 30, 065101 (2018)

34.Klein, M., Alwazzan, D., Chakraborty, N.: A Direct Numerical Simulation analysis of pressure variation in turbulent premixed Bunsen burner flames-Part 1: Scalar gradient and strain rate statistics, Computers \& Fluids, DOI: 10.1016/j.compfluid.2018.03.010 (2018)

35.Klein, M., Alwazzan, D., Chakraborty, N.: A Direct Numerical Simulation analysis of pressure variation in turbulent premixed Bunsen burner flames-Part 2: Surface Density Function transport statistics, Computers \& Fluids, DOI: 10.1016/j.compfluid.2018.03.013 (2018)

36.Haworth, D.C., and Poinsot, T.J.: Numerical simulations of Lewis number effects in turbulent premixed flames, J. Fluid Mech., 244, 405-436 (1992)

37.Rutland, C., and Trouvé, A.: Direct Simulations of premixed turbulent flames with nonunity Lewis numbers, Combust. Flame, 94, 41-57 (1993)

38.Creta, F., Lamioni, R., Lapenna, P.E., Troiani, G.: Interplay of Darrieus-Landau instability and weak turbulence in premixed flame propagation Phys. Review E, 94 ,053102 (2016)

39. Klein, M., Nachtigal, H., Hansinger, M., Pfitzner, M., Chakraborty, N.: Flame curvature distribution in high pressure turbulent Bunsen premixed flames, Flow, Turb. Combust., https://doi.org/10.1007/s10494-018-9951-1 (2018) 
40.Pope, S.B.: The evolution of surfaces in turbulence, Int. J. Eng. Sci., 26, 445-469 (1988)

41.Dopazo, C., Martin, J., Cifuentes, L. and Hierro, J.: Strain, rotation and curvature of nonmaterial propagating iso-scalar surfaces in homogeneous turbulence, Flow Turb. Combust., https://doi.org/10.1007/s10494-017-9888-9 (2018)

42.Cifuentes, L., Dopazo, C., Karichedu, A., Chakraborty, N., Kempf, A. M.: Analysis of flame curvature evolution in a turbulent premixed bluff Body burner, Phys. Fluids, 30, 095101 (2018)

43. Turns, S.R.: An introduction to combustion: concepts and applications, $3^{\text {rd }}$ Edition, McGraw Hill (2001)

44.Peters, N.: Turbulent Combustion, Cambridge Monograph on Mechanics, Cambridge University Press, Cambridge (2000)

45.Dopazo, C., Cifuentes, L., Martin, J., Jimenez, C.: Strain rates normal to approaching isoscalar surfaces in a turbulent premixed flame, Combust. Flame, 162, 1729- 1736 (2015) 46.Jenkins, K.W., Cant, R.S.: DNS of turbulent flame kernels, In C. Liu, L. Sakell and T. Beautner (Eds.), Proc. 2nd AFOSR Conf. on DNS and LES, Kluwer Academic Publishers, 192-202 (1999)

47.Chakraborty, N.; Kolla, H.; Sankaran, R.; Hawkes, E.R.; Chen, J.H.; Swaminathan, N.: Determination of three-dimensional quantities related to scalar dissipation rate and its transport from two-dimensional measurements: Direct Numerical Simulation based validation. Proc. Combust. Inst., 34, 1151-1162 (2013)

48.Dopazo, C.; Cifuentes, L.; Chakraborty, N.: Vorticity budgets in premixed combusting turbulent flows at different Lewis numbers. Phys. Fluids, 29, 045106 (2017)

49.Lipatnikov, A.N.; Nishiki, S.; Hasegawa, T.: A direct numerical study of vorticity transformation in weakly turbulent premixed flames. Phys. Fluids, 26, 105104 (2014) 
50.Gao, Y.; Chakraborty, N.; Klein, M.: Assessment of sub-grid scalar flux modelling in premixed flames for Large Eddy Simulations: A-priori Direct Numerical Simulation. Eur. J. Mech. Fluids-B, 52, 97-108 (2015)

51.Papapostolou, V.; Wacks, D.H.; Klein, M.; Chakraborty, N.; Im, H.G.: Enstrophy transport conditional on local flow topologies in different regimes of premixed turbulent combustion. Sci. Rep. 7, 11545 (2017)

52.Klein, M.; Kasten, C.; Chakraborty, N.; Mukhadiyev, N.; Im, H.G.: Turbulent scalar fluxes in Hydrogen-Air premixed flames at low and high Karlovitz numbers. Combust. Theory Model., 22, 1033-1048 (2018).

53.Gao, Y.; Chakraborty, N.: Modelling of Lewis Number dependence of Scalar dissipation rate transport for Large Eddy Simulations of turbulent premixed combustion. Numer. Heat Trans. A, 69, 1201-1222 (2016)

54.Lai, J.; Chakraborty, N.: Effects of Lewis Number on head on quenching of turbulent premixed flame: A Direct Numerical Simulation analysis. Flow Turbul. Combust., 96, 279$308(2016)$

55.Gao, Y.; Minamoto, Y.; Tanahashi, M.; Chakraborty, N.: A priori assessment of scalar dissipation rate closure for Large Eddy Simulations of turbulent premixed combustion using a detailed chemistry Direct Numerical Simulation database. Combust. Sci. Technol., 188, $1398-1423(2016)$

56.Lai, J.; Klein, M.; Chakraborty, N.: Direct Numerical Simulation of head-on quenching of statistically planar turbulent premixed methane-air flames using a detailed chemical mechanism. Flow Turbul. Combust., 101, 1073-1091 (2018)

57.Savard, A., Lapointe, S. and Teodorczyk, A.: Numerical investigation of the effect of pressure on heat release rate in iso-octane premixed turbulent flames under conditions relevant to SI engines, Proc. Combust. Inst., 36, 3543-3549 (2017). 
58.Poinsot, T., Lele, S.K.: Boundary conditions for direct simulation of compressible viscous flows, J. Comp. Phys., 101, 104-129 (1992)

59.Kobayashi, H., Kawabata, Y. and Maruta K., Experimental study on general correlation of turbulent burning velocity at high pressure, Proc. Combust. Inst., 27, 941-948 (1998)

60.Chakraborty, N., Cant, R.S.: A-Priori Analysis of the curvature and propagation terms of the Flame Surface Density transport equation for Large Eddy Simulation, Phys. Fluids, 19,105101 (2007)

61.Chakraborty, N., Cant, R.S.: Direct Numerical Simulation analysis of the Flame Surface Density transport equation in the context of Large Eddy Simulation, Proc. Combust. Inst., 32, 1445-1453 (2009)

62.Gao, Y., Chakraborty, N., Swaminathan, N.: Local strain rate and curvature dependences of scalar dissipation rate transport in turbulent premixed flames: A Direct Numerical Simulation analysis, J. Combust., 2014, 280671 (2014)

63.Gao, Y., Chakraborty, N., Swaminathan, N.: Scalar dissipation rate transport and its modelling for Large Eddy Simulations of turbulent premixed combustion, Combust. Sci. Technol., 187(3), 362-383 (2015) 